\title{
Beyond Preconditioning: Postconditioning as an Alternative Technique in the Prevention of Liver Ischemia-Reperfusion Injury
}

\author{
Kassiani Theodoraki, ${ }^{1}$ Iosifina Karmaniolou, ${ }^{2}$ Aliki Tympa, \\ Marios-Konstantinos Tasoulis, ${ }^{3}$ Constantinos Nastos, ${ }^{3}$ Ioannis Vassiliou, ${ }^{3}$ \\ Nikolaos Arkadopoulos, ${ }^{4}$ and Vassilios Smyrniotis ${ }^{4}$ \\ ${ }^{1}$ Department of Anesthesiology, Aretaieion University Hospital, University of Athens Medical School, 11528 Athens, Greece \\ ${ }^{2}$ Department of Anaesthetics, St George's Hospital, Blackshaw Road, London SW17 0QT, UK \\ ${ }^{3}$ Second Department of Surgery, Aretaieion University Hospital, University of Athens Medical School, 11528 Athens, Greece \\ ${ }^{4}$ Fourth Department of Surgery, Attikon University Hospital, University of Athens Medical School, 12462 Athens, Greece
}

Correspondence should be addressed to Kassiani Theodoraki; ktheodoraki@hotmail.com

Received 30 December 2015; Revised 11 April 2016; Accepted 5 May 2016

Academic Editor: Ersin Fadillioglu

Copyright (C) 2016 Kassiani Theodoraki et al. This is an open access article distributed under the Creative Commons Attribution License, which permits unrestricted use, distribution, and reproduction in any medium, provided the original work is properly cited.

\begin{abstract}
Liver ischemia/reperfusion injury may significantly compromise hepatic postoperative function. Various hepatoprotective methods have been improvised, aiming at attenuating IR injury. With ischemic preconditioning (IPC), the liver is conditioned with a brief ischemic period followed by reperfusion, prior to sustained ischemia. Ischemic postconditioning (IPostC), consisting of intermittent sequential interruptions of blood flow in the early phase of reperfusion, seems to be a more feasible alternative than IPC, since the onset of reperfusion is more predictable. Regarding the potential mechanisms involved, it has been postulated that the slow intermittent oxygenation through controlled reperfusion decreases the burst production of oxygen free radicals, increases antioxidant activity, suppresses neutrophil accumulation, and modulates the apoptotic cascade. Additionally, favorable effects on mitochondrial ultrastructure and function, and upregulation of the cytoprotective properties of nitric oxide, leading to preservation of sinusoidal structure and maintenance of blood flow through the hepatic circulation could also underlie the protection afforded by postconditioning. Clinical studies are required to show whether biochemical and histological improvements afforded by the reperfusion/reocclusion cycles of postconditioning during early reperfusion can be translated to a substantial clinical benefit in liver resection and transplantation settings or to highlight more aspects of its molecular mechanisms.
\end{abstract}

\section{Introduction}

Prevention of major hemorrhage during hepatic resection is crucial because of the unfavorable short- and long-term outcomes associated with blood transfusion [1]. In this context, techniques involving some type of vascular control are favored by many surgeons since they can ensure a less hemorrhagic surgical field by taking advantage of liver tolerance to normothermic warm ischemia $[2,3]$. These maneuvers, although valuable in preventing excessive blood loss, are invariably complicated by ischemia/reperfusion (IR) injury, which can reduce the capacity of the liver remnant to maintain adequate postoperative function $[4,5]$. Hepatic IR injury can also occur in other clinical contexts, such as liver donor preservation and transplantation and hypovolemia $[6,7]$. Specifically, both warm and cold ischemia, with the accompanying reduction of blood flow, cause depletion of hepatocyte energy reserves, accumulation of intracellular sodium, calcium, and reactive oxygen species (ROS), and the activation of multiple enzyme systems leading to cell damage [8]. With the restoration of blood flow through reperfusion, the liver is subjected to further injury secondary to an ensuing 
acute inflammatory response. Activated Kupffer cells, polymorphonucleocytes, and platelets infiltrate reperfused tissue, while further structural and functional disorders of hepatic tissue are mediated through abundant cytokine production, complement activation, accumulation of platelet activating factors and endothelial cell adhesion molecules, local imbalance in nitric oxide (NO) levels, and finally generation of free radicals and depletion of tissue antioxidant capacity [9-12]. The sequence of ischemia followed by reperfusion is manifested as vasoconstriction, neutrophil migration and adherence, and platelet aggregation [13-15]. The ensuing microcirculatory derangement can finally culminate in hepatocellular apoptosis and necrosis with untoward consequences not only for the liver but also for distant organs [1618].

The extent of liver parenchymal damage depends on the duration of ischemia, the presence of preexisting liver disease, and the use of hepatoprotective methods. One of the methods used to modulate IR injury is ischemic preconditioning (IPC). IPC is the method by which the target organ is conditioned with a brief ischemic period followed by reperfusion prior to the subsequent prolonged ischemic insult in order to attenuate the extent of injury. Its beneficial effects were first reported by Murry et al. in a study of canine heart tissue [19]. It has since been adopted in liver surgery and tested in several experimental and clinical contexts, proving to be an effective intervention, since it seems to increase the ability of the liver to withstand the subsequent prolonged period of ischemia [20-23]. Adenosine and NO seem to play a significant role in the IPC effect and favorable responses such as decreased hepatocellular injury, inhibition of apoptosis, improved liver microcirculation, and enhanced energy metabolism have been documented through the application of IPC [24-27].

Regarding the clinical setting, in spite of favorable effects of enzyme markers of liver injury, recent meta-analyses failed to reveal a sustained clinical benefit of IPC, in terms of duration of hospital stay, perioperative morbidity, or mortality [28-30]. The main limitation of IPC techniques in the clinical context is that they must be initiated before the ischemic insult, which is not always predictable. In recent years, a novel approach to minimize IR injury was initiated. Ischemic postconditioning (IPostC), defined as several brief cycles of ischemia and reperfusion after the prolonged period of ischemia and before the sustained reperfusion was initially described by Zhao et al. in an in vivo canine heart model [31], was proved to provide effective cardioprotection with reduction of infarct size and reperfusion arrhythmias in subsequent studies [32-35] and its favorable effects were further confirmed in a meta-analysis including 123 patients [36]. The application of the conditioning stimulus (brief intermittent cycles of IR) after prolonged ischemia and prior to permanent restoration of blood flow, as opposed to IPC, where the conditioning stimulus is applied before the prolonged period of ischemia, makes the intervention clinically more relevant, since the onset of ischemia as already mentioned cannot always be predicted. Therefore, the technique was quickly adopted in experimental liver resection and transplantation settings and in recent years there have been several reports of its use in this context.
The aim of this review was to examine the current evidence for the use of IPostC in liver resection and transplantation settings, to establish whether IPostC protects against IR injury, and to provide some insight into the potential mechanisms involved.

\section{Methods}

We conducted a systematic PubMed ${ }^{\circledR}$ literature search for all types of published articles in the English language combining the free text and MeSH thesaurus terms: "liver", "hepatectomy", "liver transplantation", "ischemia-reperfusion", "ischemia-reperfusion injury", "warm liver ischemia", "cold liver ischemia", "cold storage", and "ischemic postconditioning", "mechanical postconditioning", and "pharmacological postconditioning", in all possible combinations. Additional articles were found by manually cross-checking the references of the identified articles. In total, 25 articles pertinent to postconditioning were retrieved. Specifically, 19 studies on ischemic (mechanical) postconditioning and six studies on pharmacological postconditioning were found relevant and suitable for inclusion in the present review. Articles highlighting the specific mechanisms of postconditioning were also considered.

\section{Mechanical (Ischemic) Postconditioning}

In recent years, several experimental IR injury models have been described using various ischemic and reperfusion times in order to test the efficacy of postconditioning maneuvers (Table 1). The first study which demonstrated the protection afforded by IPostC on liver injury was reported by Sun et al., in a rat model of ischemia and reperfusion [37]. The authors established a $70 \%$ hepatic ischemia model by occluding blood flow to the left and caudate lobes of the liver. Ischemia was maintained for $60 \mathrm{~min}$, followed by a 120 -minute reperfusion period. In the conditioned group, IPostC consisted of several brief prereperfusions $(2,3,5$, and 7 min consecutively, each separated by occlusion time of $2 \mathrm{~min}$ ), followed by the sustained reperfusion. Concentrations of malondialdehyde (MDA), as an index of tissue lipid peroxidation, and activities of several antioxidant enzymes (superoxide dismutase (SOD), catalase (CAT), and glutathione peroxidase (GSH$\left.\mathrm{P}_{\mathrm{X}}\right)$ ) were measured in hepatic tissue. Additionally, apoptotic cells were measured by terminal deoxynucleotidyl transferase dUTP nick end labeling (TUNEL) staining, expression of $\mathrm{Bcl}-2$ protein (which is a major representative of apoptotic restraining proteins) was measured by immunohistochemical techniques, and mitochondrial ultrastructure was evaluated by electronic microscope. In comparison to the IR group, the concentration of MDA in the IPostC group was markedly reduced while the activity of all antioxidant enzymes and the expression of $\mathrm{Bcl}-2$ protein were significantly enhanced. Moreover, the apoptotic index in the IPostC group was significantly reduced and mitochondrial ultrastructure was maintained basically normal, with attenuation of mitochondrial swelling and maintenance of the integrity of outer mitochondrial membrane. In a similar model of controlled reperfusion, Zhang et al. additionally measured alanine aminotransferase 


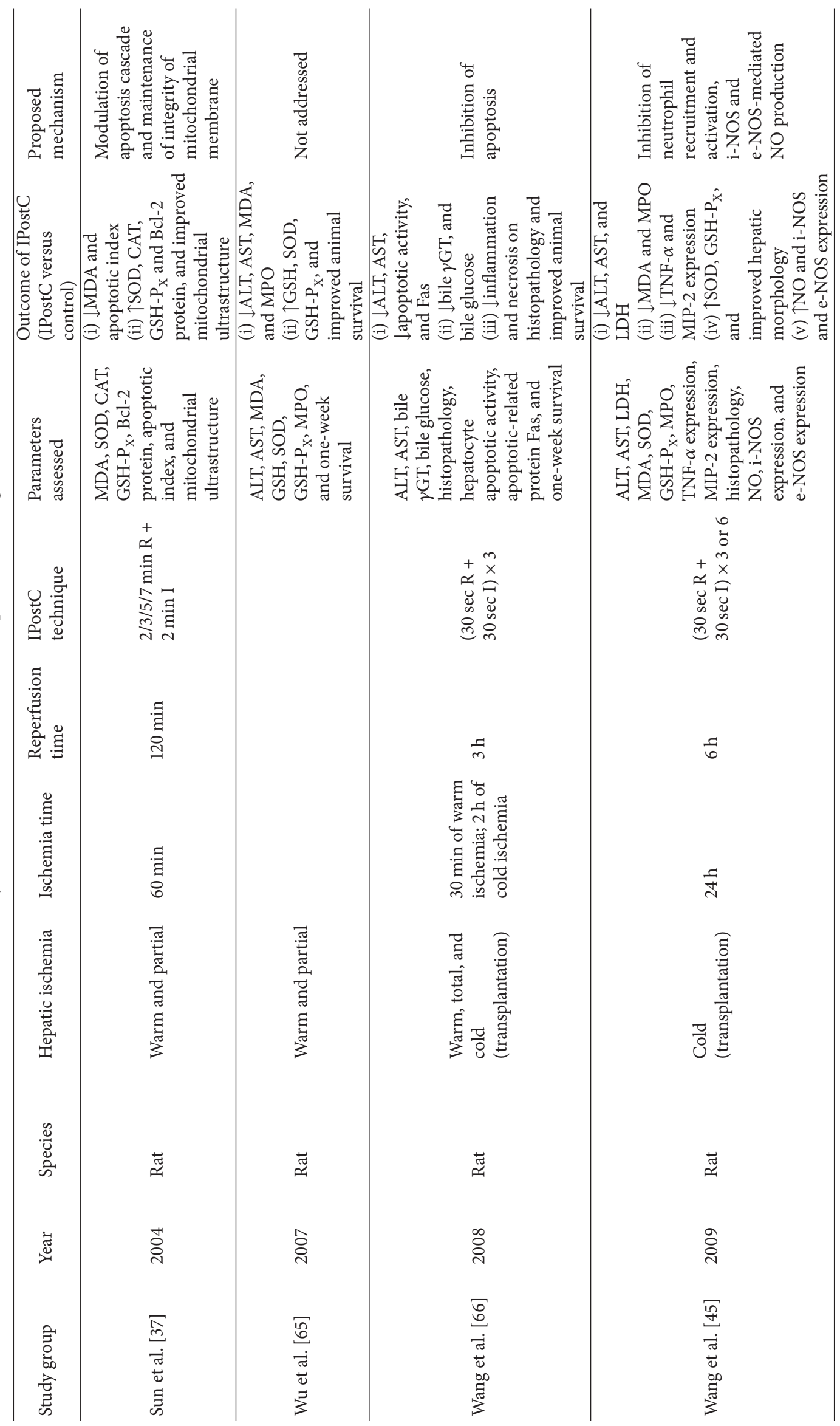




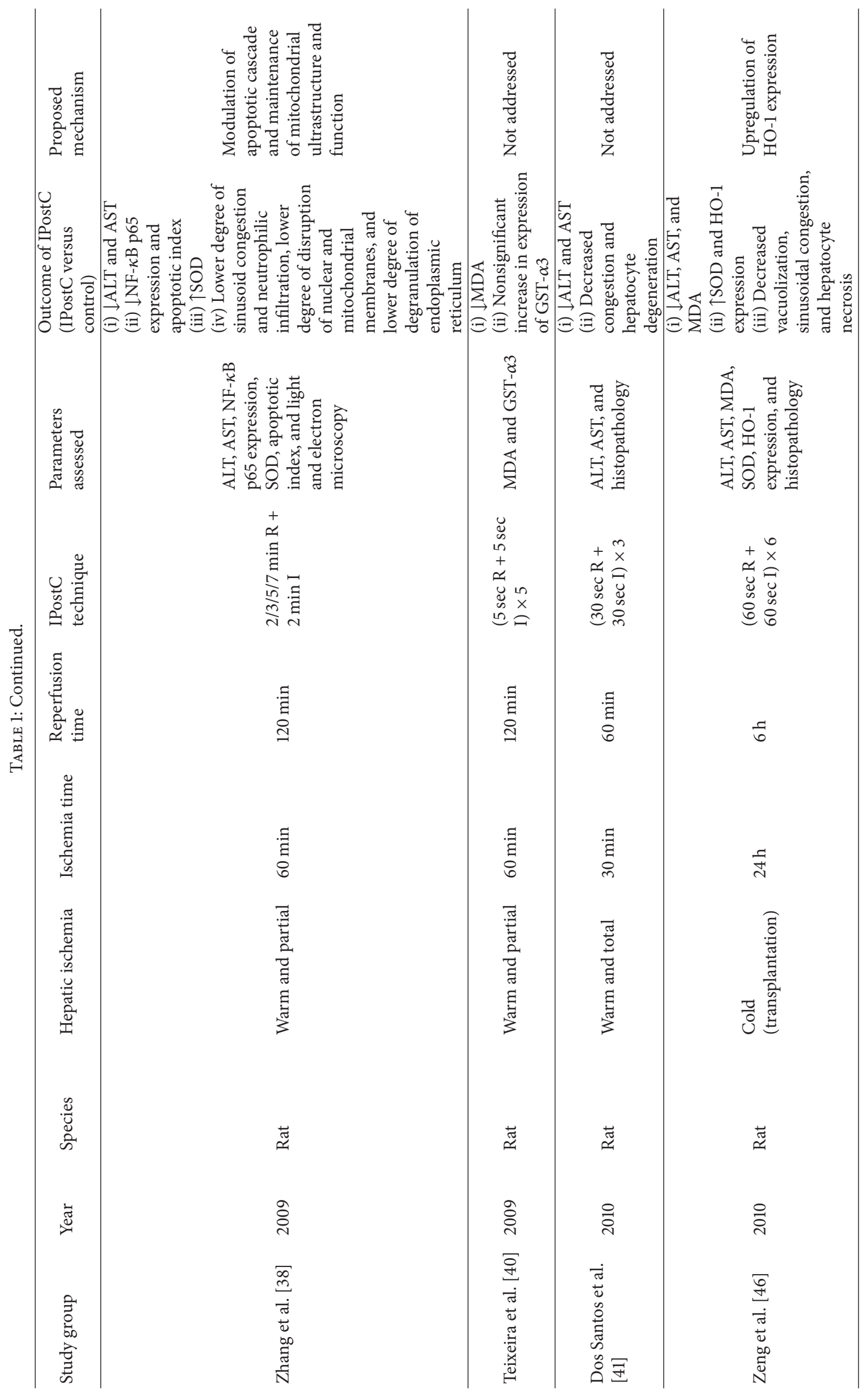




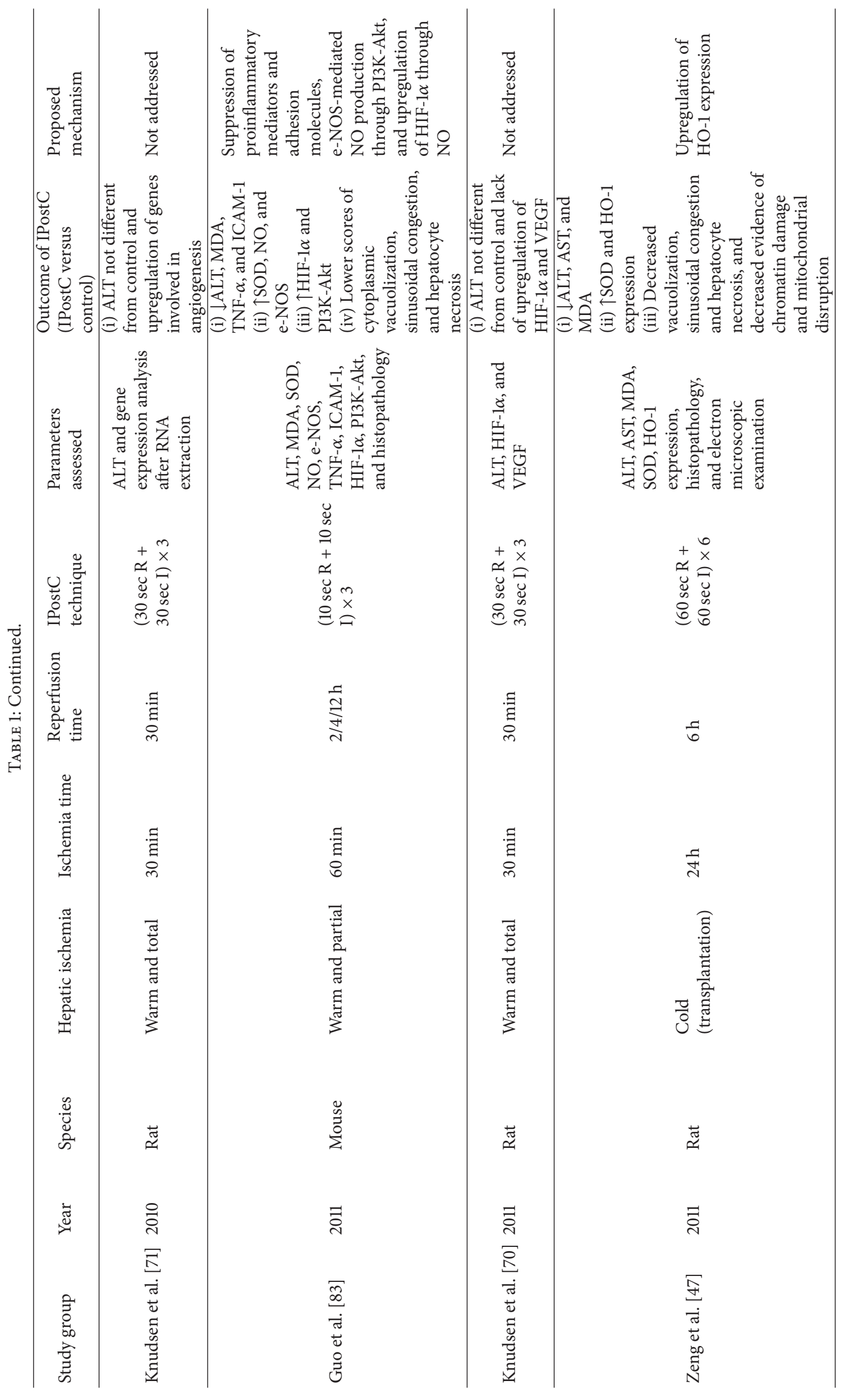




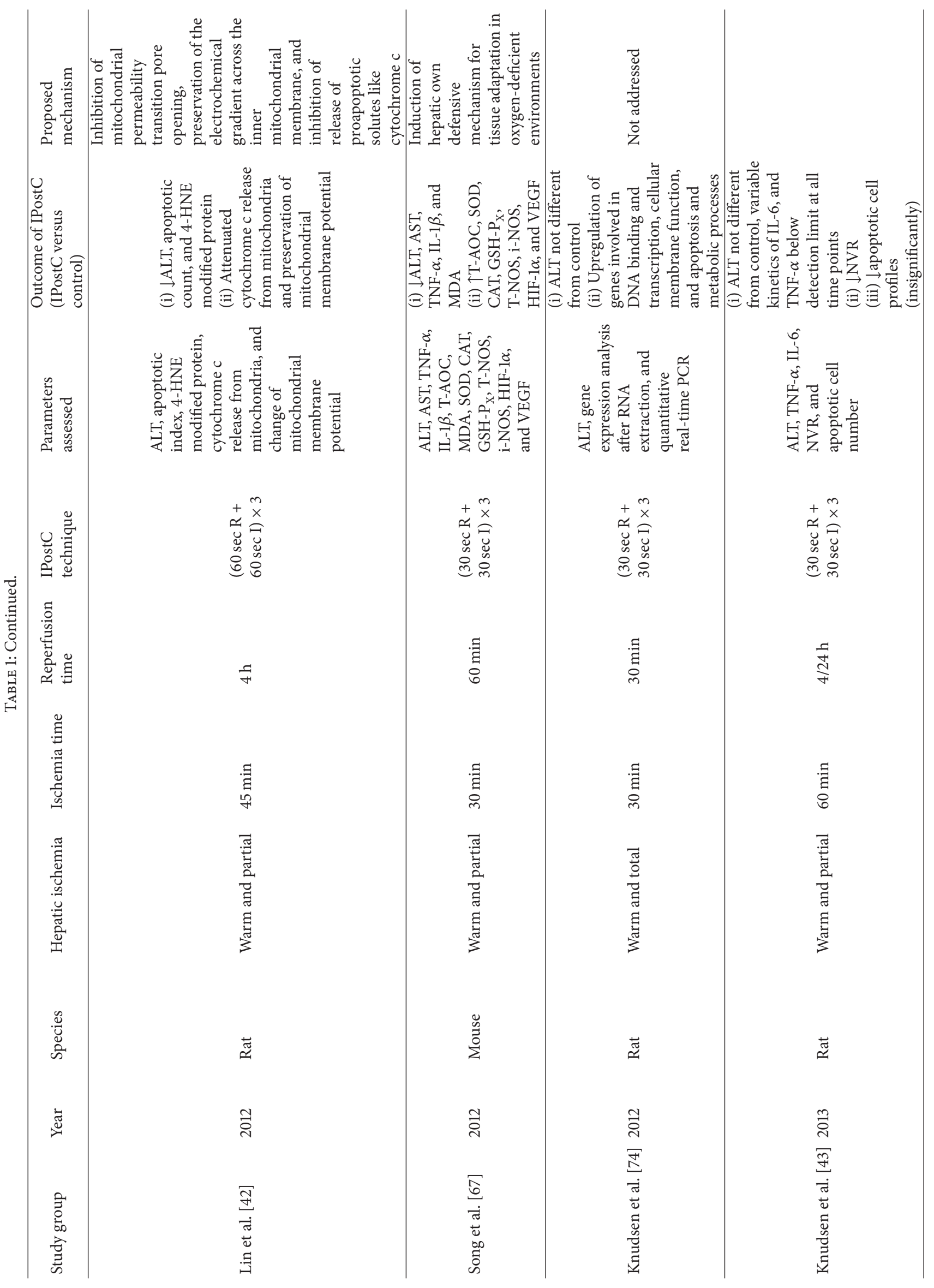




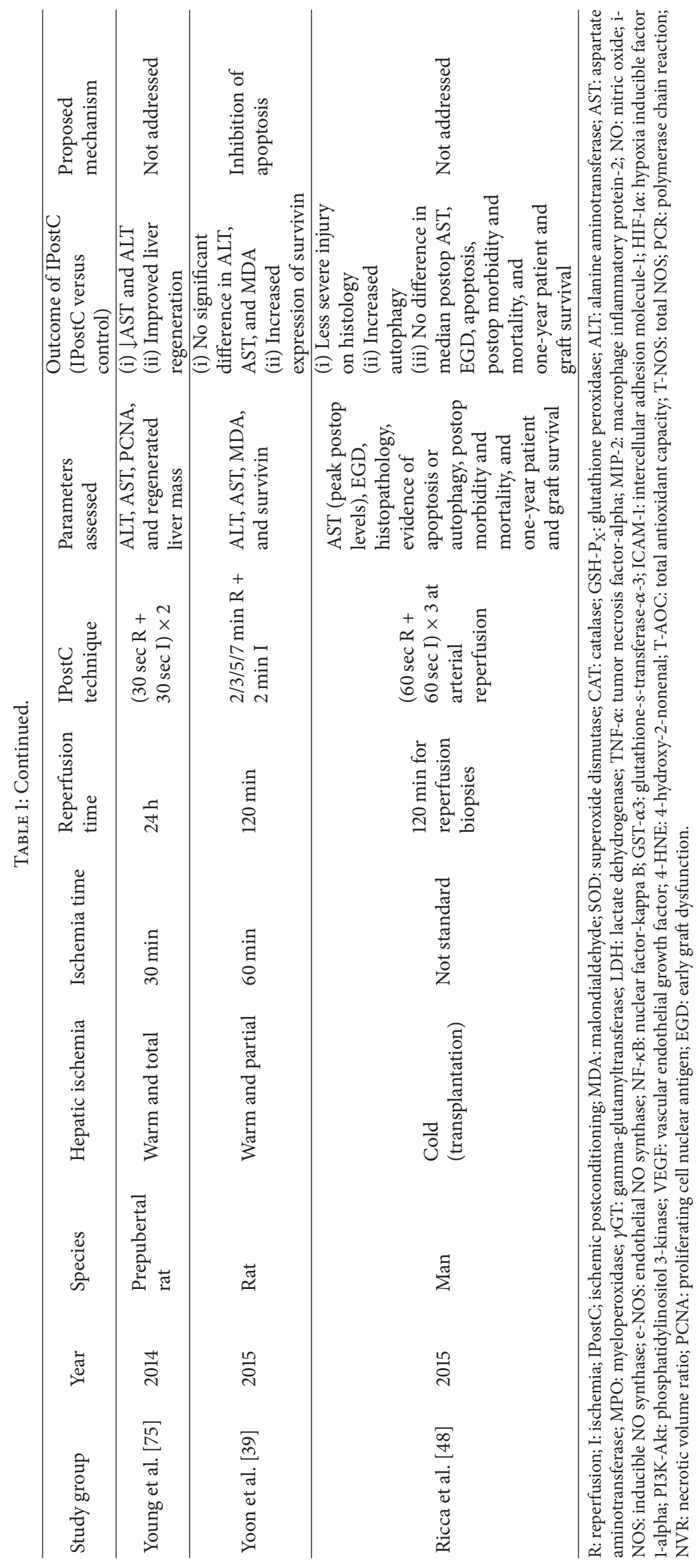


(ALT) and aspartate aminotransferase (AST) as well as nuclear factor-kappa beta (NF- $\kappa \mathrm{B}$ ) p65 (whose generation is activated by a large amount of oxygen free radicals) and also found lower levels of ALT and AST, increased SOD activity, and reduced NF- $\kappa \mathrm{B}$ p65 expression and apoptotic index in the IPostC group in comparison to the IR group [38]. They also observed differences in light and electron microscopy, with a lower degree of congestion of hepatic sinusoids, reduced neutrophilic infiltration, and fewer cells with disruption of nuclear and mitochondrial membranes or degranulation of endoplasmic reticulum in the IPostC group, as compared to the IR group. The authors attributed the protection afforded by postconditioning to the controlled slow intermittent oxygenation through the several cycles of on/off flow before permanent reperfusion. Finally Yoon et al., adopting the same sequence of on/off flow before permanent reperfusion, showed increased expression of the antiapoptotic protein survivin in the IPostC group in comparison to the IR group [39].

Different protocols of postconditioning have been used by other authors. Teixeira et al., using a rapid intermittent reperfusion consisting of five periods of $5 \mathrm{sec}$ of opening followed by $5 \mathrm{sec}$ of clamping each before the prolonged reperfusion, demonstrated decreased lipid peroxidation assessed by MDA levels and enhanced expression (although not significantly) of the glutathione-s-transferase- $\alpha-3$ gene, assessed by realtime RNA analysis [40]. Also, by interposing three cycles of reperfusion followed by three cycles of ischemia (lasting $30 \mathrm{sec}$ each) between ischemia and prolonged reperfusion, another group of researchers showed attenuated liver injury assessed by serum AST and ALT levels and attenuated histological scores of hepatic lesion in the postconditioned group [41]. Lin et al., after generating a 45-minute period of left lobe ischemia, performed three cycles of 1-minute reperfusion followed by $1 \mathrm{~min}$ of ischemia before sustained reperfusion and measured serum ALT levels and the degree of apoptosis by TUNEL staining and the formation of 4-hydroxy-2-nonenal (4-HNE) modified protein, which is generated during the lipid peroxidation cascade and is used as a biomarker of oxidative stress. They also evaluated cytochrome $\mathrm{c}$ release by mitochondria with Western blot analysis and change of mitochondrial membrane potential through flow cytometry analysis [42]. In comparison to the IR group, IPostC attenuated the elevation of ALT as well as the apoptotic count and decreased lipid peroxidation, as assessed by decreased expression of 4 -HNE protein. IPostC also effectively reduced cytochrome $c$ release from mitochondria and preserved mitochondrial membrane potential as compared to nonconditioned animals. Based on the notion that the opening of mitochondrial permeability transition pores (mPTP) with the resultant release of cytochrome $\mathrm{c}$ and collapse of transmembrane potential plays a crucial role in the pathogenesis of reperfusion injury, the authors concluded that the hepatoprotective effects of postconditioning are mediated through modulation of mitochondrial permeability transition.

Most of the studies mentioning histological results have based their conclusions on semiquantitative histopathologic evaluations. A recent study used stereological methods to obtain quantitative three-dimensional histological information in order to assess the effect of IPostC on the liver [43]. In this report, Knudsen et al. focused on necrosis and apoptosis which are irreversible signs of hepatocellular injury in contrast to vacuolization and sinusoidal congestion, which may be reversible, and additionally based their results on design-based stereology, which is an objective and highly reproducible method [44]. The authors established their ischemic model by interrupting portal trial flow to the median and left lobes for $60 \mathrm{~min}$ after which they evaluated a 4- or 24-hour reperfusion period. The IPostC protocol consisted of three cycles of $30 \mathrm{sec}$ of reperfusion and $30 \mathrm{sec}$ of ischemia performed immediately after the $60 \mathrm{~min}$ of prolonged ischemia. Although no significant differences were demonstrated after four hours of reperfusion, at 24 hours of reperfusion, the authors observed a significant decrease in necrotic volume ratio (NVR) and an insignificant decrease of apoptotic cell profiles in the IPostC group in comparison to the control group.

IPostC has also been evaluated in the experimental context of liver transplantation. In a rat model of orthotopic liver transplantation, Wang et al. applied postconditioning after cold ischemia of the donor liver using several intermittent interruptions of blood flow at the early phase of reperfusion and compared grafts treated with IPostC with controls, after six hours of sustained reperfusion of the liver graft [45]. Apart from standard histopathologic examination, they evaluated serum parameters of hepatocellular injury (AST, ALT, and lactate dehydrogenase (LDH)), lipid peroxidation (MDA), antioxidant enzyme activity in liver tissue ( $\mathrm{SOD}, \mathrm{GSH}-\mathrm{P}_{\mathrm{X}}$ ), and myeloperoxidase (MPO) as a marker of polymorphonuclear neutrophil infiltration. They also used reverse transcriptase-polymerase chain reaction (RT-PCR) RNA analysis to quantify the expression of tumor necrosis factor-alpha (TNF- $\alpha$ ) and macrophage inflammatory protein-2 (MIP-2) in liver tissue. They additionally measured NO content in serum and the expression of inducible NO synthase (i-NOS) and endothelial NO synthase (e-NOS) in liver tissue. Markers of hepatocellular injury were markedly reduced when grafts were treated with postconditioning, while IPostC inhibited lipid peroxidation, enhanced antioxidant enzyme activity, suppressed polymorphonuclear accumulation, lowered the expression of TNF- $\alpha$ and MIP2 , and reduced the extent of necrosis in histopathologic examination. Furthermore, increases in serum NO and iNOS and e-NOS expression were much more prominent when grafts were treated with postconditioning. The authors postulated that i-NOS- and e-NOS-mediated endogenous NO production might also underlie the protection afforded by IPostC. Zeng et al. also demonstrated that IPostC attenuated liver IR injury in a cold ischemia model, since it attenuated serum transaminase levels and histological damage in postconditioned grafts, while it reduced MDA production and increased SOD activity in comparison to the IR group [46]. They implicated induction of the cytoprotective enzyme heme oxygenase-1 (HO-1) in the enhancement of antioxidative activity associated with postconditioning. They reinforced their findings in a subsequent study, where, by pretreating donors with an inhibitor of HO-1 before liver 
harvest, they demonstrated negation of the protective effects of IPostC [47].

The only clinical study on IPostC has just recently been reported [48]. The authors attempted to test the role of IPostC in human liver transplantation and its effects on IR injury and liver graft function. IPostC consisted of three 1-minute cycles of reperfusion interspersed with three 1-minute cycles of arterial occlusion immediately after arterial reperfusion of the graft. Median postoperative peak AST values, indices of early graft dysfunction, apoptosis, morbidity (including graft rejection), early postoperative mortality, and one-year patient and graft survival were similar between patients subjected to IPostC and the control group. However, grafts subjected to postconditioning presented less severe histopathological lesions of IR injury and increased activation of autophagy in periportal areas. Autophagy is triggered by stress conditions to ensure cell survival by restoring adequate levels of intracellular ATP, a fact that has been correlated with better posttransplantation outcomes $[49,50]$. Therefore, the study results showed that grafts subjected to IPostC had better tolerance of IR injury according to histological parameters, which, together with the induction of autophagy, is indicative of the restoration of sufficient energy reserves in injured hepatocytes.

\section{Pharmacological Postconditioning}

Apart from mechanical postconditioning, there have been studies showing that some agents can be used as pharmacological inducers of postconditioning. The administration of agents upon liver reperfusion aiming at mitigating IR injury is defined as pharmacological postconditioning (Table 2). Milrinone, a phosphodiesterase-3 inhibitor, is an inotropic agent, acting through elevation of intracellular cyclic adenosine monophosphate (cAMP) and protein kinase A (PKA) activation. It has been shown that it also has preconditioning properties against hepatic IR injury, exerted via the same pathway (cAMP/PKA activation) [51]. Toyoda et al., in a liver warm ischemia model of one-hour duration followed by five hours of reperfusion, showed that milrinone administered as an intravenous bolus immediately after reperfusion effectively attenuated liver injury, as demonstrated by reduced AST, ALT, and LDH serum levels and reduced histologic damage and apoptotic scores in milrinone-treated animals, as compared to controls [52]. The authors postulated that the protective effect of milrinone could be mediated through phosphatidylinositol 3-kinase (PI3K-Akt) and NOS activation, as the beneficial effects of milrinone were abrogated by inhibition of PI3K-Akt and NOS.

Tian et al. administered diazoxide (a selective ATPdependent mitochondrial potassium (mito- $\mathrm{K}_{\mathrm{ATP}}$ ) channel opener) before reperfusion, in a warm liver ischemia rat model [53]. Diazoxide treatment significantly attenuated hepatic injury, as demonstrated by serum levels of AST and ALT, while it upregulated levels of protein kinase c-epsilon (pkc-e), which is a kinase necessary for the opening of mito- $\mathrm{K}_{\mathrm{ATP}}$ channels that are involved in the protection from reperfusion injury $[54,55]$. Additionally, diazoxide inhibited the activation of the apoptotic pathway by increasing the expression of apoptotic restraining protein $\mathrm{Bcl}-2$ and by decreasing the release of cytochrome $\mathrm{c}$ and the expression of caspase-3.

Beck-Schimmer et al. designed the only known clinical study of pharmacological hepatic postconditioning, using the anesthetic agent sevoflurane [56]. They evaluated whether pharmacological postconditioning with sevoflurane confers protection during liver surgery under inflow occlusion compared with control and whether pharmacological postconditioning with sevoflurane confers equivalent protection to the technique of intermittent clamping during ischemia. All patients were anesthetized with propofol. In the postconditioning group, sevoflurane was administered for a 30 -minute period upon reperfusion of the liver, replacing propofol infusion. The postconditioning group displayed lower peak AST values within the first seven postoperative days as well as shorter hospital stay and a reduced risk of complications in comparison to the control group. No significant differences were demonstrated between the postconditioning and intermittent clamping groups, indicating a similar degree of protection. The same group, in a previous study, had demonstrated that pharmacological preconditioning with sevoflurane provided hepatoprotection in patients undergoing major liver resection [57]. The importance of these studies lies not only in the fact that they were performed on human subjects but also in the fact that reduced laboratory indices of liver injury were accompanied by improvements in clinical outcome.

Dal Ponte et al. investigated whether an adenosine $\mathrm{A}_{2} \mathrm{~A}$ receptor agonist could act as a pharmacological inducer of postconditioning [58]. They set up an in vitro experimental model of freshly isolated rat hepatocytes mimicking hepatocyte reoxygenation injury after the cold ischemia phase of liver graft preservation. The addition of the $\mathrm{A}_{2} \mathrm{~A}$ receptor agonist significantly reduced hepatocyte death upon reoxygenation through a PI3-Akt-mediated response. They further confirmed their results in an in vivo model of warm IR injury, where rats exposed to intraperitoneal injection of the $\mathrm{A}_{2} \mathrm{~A}$ receptor agonist immediately upon reperfusion presented with reduced ALT release and fewer necrotic areas on histological examination, as compared to controls. The authors concluded that adenosine $\mathrm{A}_{2} \mathrm{~A}$ receptor stimulation effectively elicits postconditioning responses in liver cells through modulation of PI3-Akt-dependent signaling and is in agreement with studies that have shown that the stimulation of the same receptors triggers hepatic preconditioning and prevents cell death $[59,60]$.

Ginsenoside $\mathrm{Rb} 1 \mathrm{Rb} 1$ ) is the effective ingredient of ginseng root, a root with known antioxidant properties. There have been reports of favorable effects of Rbl on liver injury induced by intestinal IR or tert-butyl hydroperoxide $[61,62]$. Guo et al. demonstrated its postconditioning effects in a mouse warm liver ischemia model [63]. They showed reduced serum ALT levels and lower scores of cytoplasmic vacuolization, sinusoidal congestion, and hepatocyte necrosis on histological examination as well as suppression of the overexpression of proinflammatory mediators and adhesion molecules, decreased concentration of MDA, and increased activity of SOD in hepatic tissues of Rbl-postconditioned 


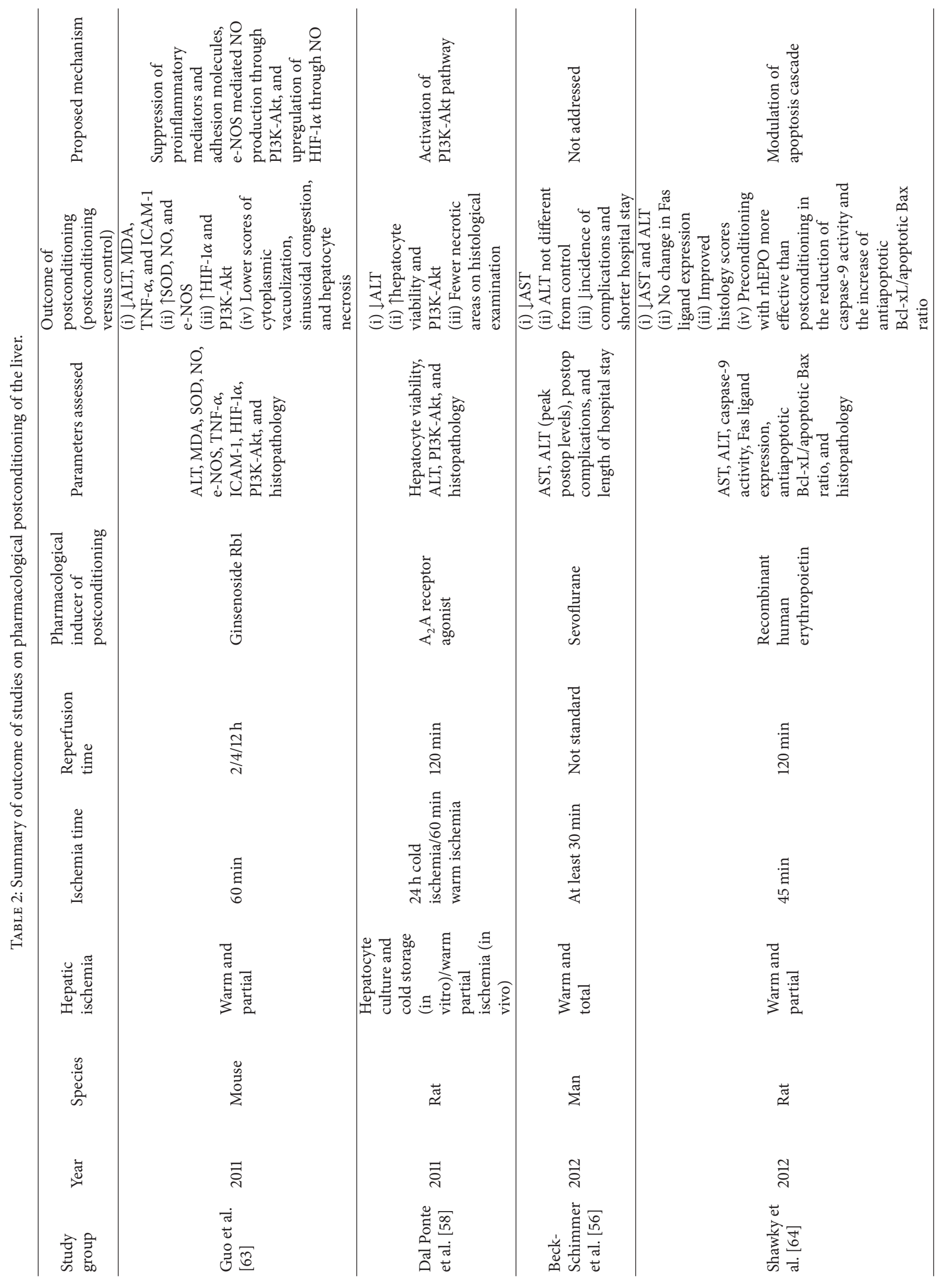




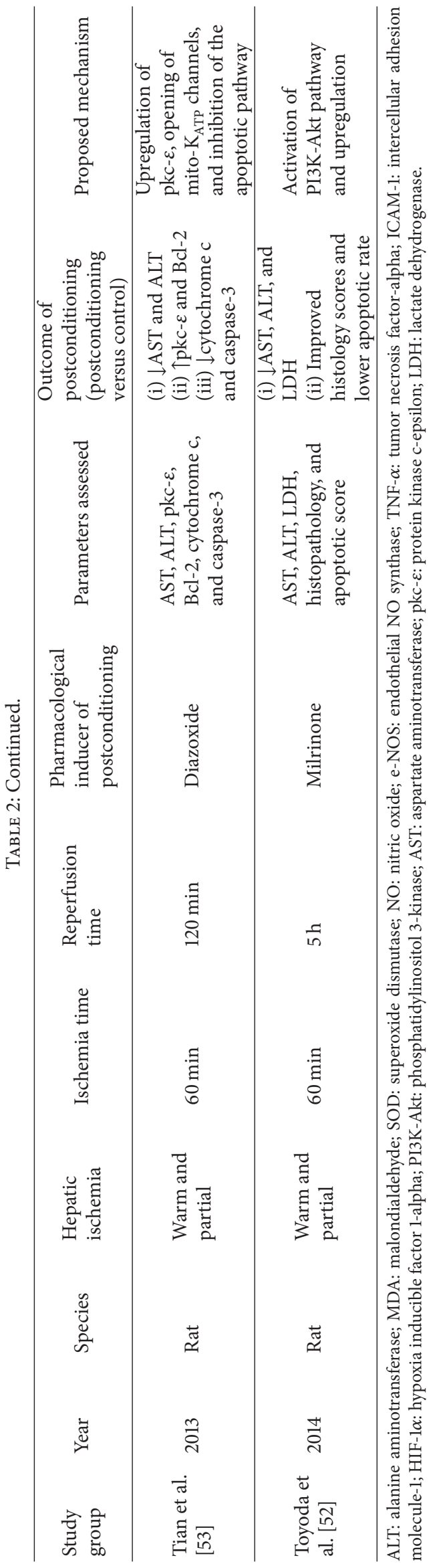


animals. They suggested that the protection conferred by Rb1 appears to be NO-mediated, as serum levels of NO and NOS and expression of NOS in liver tissues were also increased compared to controls.

Finally, Shawky et al. investigated the effect of intraportal recombinant human erythropoietin (rhEPO) in a rat model of hepatic IR injury as well as its appropriate time and dose of administration [64]. Moreover, they compared preconditioning with rhEPO ( $24 \mathrm{~h}$ or $30 \mathrm{~min}$ before ischemia) with postconditioning with rhEPO (administering it at reperfusion). Both preconditioning and postconditioning with rhEPO were effective in attenuating hepatic injury, as assessed by decreased AST and ALT serum levels while preconditioning was more effective than postconditioning in attenuating IRinduced apoptosis, as assessed by the reduction of caspase9 activity and the increase of antiapoptotic $\mathrm{Bcl}-\mathrm{xL} /$ apoptotic Bax ratio.

\section{Comparison between Postconditioning and Preconditioning}

A few comparative studies between IPC and IPostC have also been undertaken. Zhang et al. demonstrated equal protection by IPC and IPostC in terms of AST, ALT, and SOD activity, apoptotic index, and light and electron microscopy findings [38]. Wu et al., using a rat model of segmental IR injury, showed that the level of protection afforded by postconditioning is comparable to that afforded by preconditioning regarding production of ROS, maintenance of the activity of the antioxidant systems, suppression of neutrophil recruitment, and animal survival [65]. A comparative study between IPC and IPostC has also been reported by Wang et al. [66]. The authors established a warm IR hepatic model, by clamping the hepatic pedicle for $30 \mathrm{~min}$, and a cold IR model, by performing orthotopic liver transplantation within two hours of cold storage. IPC and IPostC provided an equal level of protection in terms of serum transaminase levels, biliary epithelial cell function, hepatic morphology, survival rate, and the expression of Fas apoptotic gene. Interestingly, in the cold ischemic model, IPostC was more effective than IPC in inhibiting apoptosis, as expressed by a lower apoptotic index. In a murine warm hepatic IR model, Song et al. demonstrated that pre- and postconditioning protocols were equally effective in reducing liver injury as assessed by reduction of AST and ALT levels, suppression of cytokine and MDA levels, and increase of the activity of antioxidant enzymes (SOD, CAT, GSH-P ${ }_{X}$, and NOS) [67]. Additionally, both IPC and IPostC upregulated the expression of hypoxia inducible factor 1-alpha (HIF- $\alpha$ ) and of vascular endothelial growth factor (VEGF). HIF-1 $\alpha$ is a master transcriptional factor activated by low oxygen tension that facilitates cellular adaptation to hypoxia in oxygen-deficient environments [68] and it can activate other genes, such as VEGF. VEGF acts on endothelial cell proliferation, migration, and cell organization during recovery phases after hepatic microvascular dysfunction, promoting the secretion of growth and survival factors [69]. In the study by Knudsen et al., who used designbased stereology to obtain quantitative three-dimensional histological information, both IPC and IPostC were equally effective in preventing hepatocellular necrosis, as assessed by NVR, while a significantly lower number of apoptotic cell profiles were achieved only in the IPC group [43]. In contrast, the number of apoptotic cell profiles decreased insignificantly in the IPostC group. Finally, Shawky et al. compared preconditioning and postconditioning with rhEPO, administered before ischemia and immediately after reperfusion, respectively [64]. As already mentioned, they demonstrated that preconditioning was more effective than postconditioning in attenuating IR-induced apoptosis, as assessed by the reduction of caspase- 9 activity and the increase of antiapoptotic $\mathrm{Bcl}-\mathrm{xL}$ /apoptotic Bax ratio.

\section{Combination of Preconditioning and Postconditioning}

IPC and IPostC have been combined in a couple of studies. Song et al. tested the combination of IPC and IPostC in the murine warm hepatic IR model described previously [67]. The combination of IPC and IPostC offered synergistic protection in comparison to IPC or IPostC alone, decreasing AST and ALT levels and increasing the activity of antioxidants, of hypoxia tolerance response, and of the speed of cell proliferation at a greater extent as compared to the individual treatments. However, the combination had no additional favorable effect on cytokine release as compared to solo treatment, probably because, by individual application of IPC and IPostC, the limit of decreased cytokine release is reached, as suggested by the authors [67]. The second study in which the combination of IPC and IPostC was evaluated was the work by Wu et al. [65]. The authors demonstrated equal protection of the combined treatment in comparison to IPC or IPostC alone on the production of ROS, the maintenance of the activity of the antioxidant systems, and the suppression of neutrophil recruitment. However, in that study, no additive effect from the combined treatment on the reduction of liver IR injury was demonstrated.

\section{Postconditioning and Liver Regeneration}

In the study by Song et al. [67], both IPC and IPostC upregulated the expression of HIF- $1 \alpha$ and of VEGF, which is a factor crucial for endothelial cell proliferation and organization during tissue recovery phases, as mentioned above. However, in another study, HIF- $1 \alpha$ did not prove to be a mediator of the cytoprotective effects of either preconditioning or postconditioning, since HIF- $1 \alpha$ mRNA expression was lower in all conditioned groups in comparison to the IR group [70]. Similarly, conditioning did not upregulate the expression of VEGF. In the same study, no significant differences in ALT serum levels were demonstrated between conditioned groups and the IR group. However, as the authors themselves acknowledge, these findings could be explained by the fact that they used a very short period of ischemia and reperfusion (30 min resp.), which might not be adequate to demonstrate the full extent of IR injuries. Therefore, they were unable to see any hepatoprotective effects of conditioning, as assessed 
by changes in liver parameters, as these have been demonstrated in other studies. The very short period of follow-up after reperfusion could also explain the low levels of HIF-1 $\alpha$ and VEGF in preconditioned and postconditioned animals in their model, as the 30 minutes of reperfusion studied and the lack of following expression levels over time probably did not allow the full effect of changing HIF-1 $\alpha$ and VEGF levels to develop, according to the authors [70]. Additionally, Knudsen et al. studied the effect of ischemic pre- and postconditioning on the expression of genes with angiogenic potential in rat liver [71]. In the conditioned groups, genes involved in angiogenesis were significantly upregulated. Therefore, both preconditioning and postconditioning seem to be potent activators of angiogenic genes. However, as the authors themselves state, this might prove a double-edged sword, since it may prove favorable for the regenerating liver but on the other hand it might stimulate the growth of micrometastases $[72,73]$. The same group of authors, in a subsequent study aiming to investigate the genomic response induced by IPC or IPostC, used the same experimental protocol and validated their microarray analysis by performing quantitative realtime PCR [74]. They found that a substantial number of genes, especially those involved in DNA binding and transcription, cellular membrane function, apoptosis, and metabolic processes are affected, especially by postconditioning or by the combination of preconditioning and postconditioning. This indicates that conditioning techniques might mediate their protective effect during the early reperfusion phase by activating the expression of gene networks crucial to cellular growth, proliferation, repair, and homeostasis. Overall, upregulated pathways seem to increase the cellular resistance to stressful conditions and the defense of the rat liver against IR injuries.

Finally, the most recent study investigating the effect of postconditioning or preconditioning on liver regeneration is by Young et al., who subjected prepubertal rats to total hepatic ischemia for $30 \mathrm{~min}$ through hepatic pedicle clamping and then to 24-hour reperfusion [75]. IPC consisted of 10-minute ischemia followed by 10 -minute reperfusion before the main ischemic event and IPostC consisted of two 30-second reperfusion/clamping cycles before sustained reperfusion. The authors measured AST and ALT serum levels, proliferating cell nuclear antigen (PCNA) with immunohistochemistry, and evaluated liver regeneration with the calculation of regenerated liver mass according to a special formula. They found that both IPC and IPostC attenuated AST and ALT levels and thus protected the liver of growing rats against IR injury and that, interestingly, IPostC was more effective than IPC in terms of liver regeneration. This is in contrast with a couple of studies which demonstrated increased cell proliferation in models of total ischemia and partial hepatectomy with IPC; however both these studies used extracorporeal shunts and probably this should be taken into account [76, 77]. As the authors state, their findings could prove of great interest in the context of split-liver transplantation or living donor liver transplantation, where there is subsequent need for regeneration of both donor remnant and recipient graft liver and therefore the protection afforded by IPostC could offer an additional advantage.

\section{Mechanisms of Hepatic Postconditioning}

The exact mechanism through which postconditioning exerts its protective action on liver tissue has not been elucidated yet. It has been postulated that the slow intermittent oxygenation, when controlled reperfusion is applied through postconditioning, decreases the burst production of oxygen free radicals that accumulate with abrupt oxygenation of the liver cell and thus stimulates the release of intracellular antioxidant enzymes and free radical scavengers that convey hepatoprotection. Lower levels of lipid peroxidation substances, as expressed by MDA, and higher levels of antioxidant enzymes induced by IPostC in the aforementioned studies show its ability to act as a line of defense against oxidative injury in tissues [37, 38, 45-47, 65, 67]. MDA is a cytotoxic reactive aldehyde, which is formed when cell membranes are degraded by ROS; therefore it is considered as a biomarker of oxidative stress [78]. Pharmacological postconditioning also may reduce oxidative stress by attenuating the increase of MDA and increasing antioxidant activity [63].

Although the enormous generation of ROS during reperfusion plays an important role in reperfusion injury, low levels of ROS have been paradoxically implicated as an essential transduction component in protective pathways, upregulating the endogenous antioxidant enzyme activities, a fact referred to as "redox signaling." Therefore, moderate concentrations of ROS during reperfusion might also confer a beneficial effect and ROS signaling at early reperfusion has been shown to mediate protective effects of both IPC and IPostC in heart tissue $[79,80]$. It could thus be possible that one of the triggers of hepatic postconditioning protection could be the ROS availability during early reperfusion, which might contribute to the activation of protective intrinsic mechanisms against the deleterious effects of the subsequent reperfusion.

Additionally, it has been postulated that cell apoptosis could also be a primary mechanism of the damage evoked by liver IR injury $[17,18]$. Modulation of the apoptotic cascade has been demonstrated in a variety of postconditioning studies and together with downregulation of the release of proapoptotic solutes could be one aspect of the protection afforded by IPostC $[37,38,42,66]$. Pharmacological inducers of postconditioning were also found to inhibit the activation of the apoptotic pathway $[52,53,64]$. In relation to oxidantinduced apoptosis, the role of NF- $\kappa \mathrm{B}$ has also been investigated. NF- $\kappa \mathrm{B}$ is a transcriptional factor whose generation is activated by a large amount of oxygen free radicals [81]. It has been shown that oxidant-induced apoptosis after myocardial ischemia and reperfusion can be activated through translocation of NF- $\kappa$ B and stimulation of the release of TNF- $\alpha$ while application of postconditioning at the onset of reperfusion attenuated myocardial apoptosis through inhibition of NF- $\kappa \mathrm{B}$ translocation [82]. Reduced NF- $\kappa$ B p 65 expression along with apoptotic index in the IPostC group in comparison to the IR group was demonstrated in the Zhang study [38]. Therefore, hepatic postconditioning could also be associated with the inhibition of oxidant-mediated activation of nuclear factor $\kappa \mathrm{B}-\mathrm{TNF}-\alpha$ signaling pathway. 
Apart from improving activity of endogenous antioxidant enzymes, postconditioning could exert its protective effect by inhibiting neutrophil accumulation, as IPostC has been shown to suppress neutrophil infiltration in hepatic tissues as well as chemokines that play a crucial role in neutrophil recruitment and activation, like MIP-2 [45]. IPostC has also been demonstrated to suppress the expression of intercellular adhesion molecule-1 (ICAM-1) in liver tissue [83], while suppression of ICAM-1 has also been demonstrated by $\mathrm{Rb} 1$ postconditioning [63]. ICAM-1 is one of cell-surface adhesion molecules that are known to mediate leukocyte-endothelial cell interaction. This molecule is present at low levels on most endothelial cells and is upregulated in case of inflammation and IR injury [84]. In fact, blocking its activity with monoclonal antibodies has been found to protect against IR injury $[85,86]$. Accumulated polymorphonucleocytes act as important effector cells in the pathogenesis of IR liver injury. During ischemia, neutrophils accumulate in the endothelium and such accumulation may be markedly accelerated following reperfusion. Activated neutrophils release a variety of cytotoxic substances interacting with the endothelium and thereby causing tissue damage and releasing large amounts of ROS, which contribute to the oxidative injury associated with hepatic IR injury $[87,88]$. Furthermore, it has been shown that Kupffer cell stimulation might lead to excessive production of TNF- $\alpha$ at the initial phase of reperfusion [89]. TNF- $\alpha$ could stimulate chemokine production like MIP-2 in hepatocytes of prolonged IR injury, which in turn facilitates polymorphonuclear activation [90]. It could thus be possible that postconditioning might protect from reperfusion injury through downregulation of substances that mediate neutrophil adhesion and activation like TNF- $\alpha$, MIP-2, and ICAM-1, as it has been shown in some of the aforementioned studies $[45,63,83]$.

It has also been suggested that the hepatoprotective effects of postconditioning are mediated through favorable effects on mitochondrial ultrastructure and function [37, 38, 42]. It has been postulated that the burst of oxygen free radicals generated by reperfusion reacts with unsaturated fatty acids on the surface of mitochondrial membrane, leading to opening of mitochondrial permeability transition pores (mPTP) [91]. When these pores open, the electrochemical gradient across the inner mitochondrial membrane is disrupted, which results in swelling and rupture of the outer mitochondrial membrane [92]. Release of apoptosis-related substances originally located in the mitochondria including cytochrome c ensues, which then transfer into the cytoplasm and subsequently activate the downstream cascade apoptosis reaction, while at the same time playing a key element in cell death [93-96]. Administration of mitochondrial permeability transition inhibitors has been shown to mitigate reperfusion injury after experimental liver transplantation [97]. Targeting mitochondrial dysfunction and inhibition of mPTP could also underlie the hepatoprotective mechanism of postconditioning. In fact, in IPostC studies, electron microscopic imaging has revealed attenuation of mitochondrial damage and basically intact mitochondrial membranes in animals subjected to the slow controlled reperfusion through postconditioning, while mitochondrial swelling and disrupted mitochondrial membranes were evident in livers not subjected to postconditioning [37, 38, 47]. In these studies, manifestations of apoptosis like condensation of chromatin into clumps at the edge of the nucleus and swelling and rounding of cells were also evident by electron microscopic observation. Additionally, Lin et al. demonstrated effective reduction of cytochrome $c$ release from mitochondria by IPostC as well as preservation of mitochondrial membrane potential [42]. Decreased expression levels of cytochrome c in hepatic tissue were also demonstrated when diazoxide was used as a pharmacological inducer of postconditioning [53]. Therefore, inhibition of mPTP opening could be associated with the cytoprotective effects of postconditioning [98-100]. However, the real trigger that inhibits mPTP opening by postconditioning is not known. It has been postulated by Cohen et al. that IPostC maintains intracellular acidosis at the initial stage of reperfusion and that perpetuation of acidosis inhibits mPTP opening in cardiac IR models [101]. The $\mathrm{pH}$ hypothesis though and its applicability in liver tissue need to be validated by further studies. With relevance to the mitochondria, the opening of mito- $\mathrm{K}_{\text {ATP }}$ channels could also underlie the protection of hepatic postconditioning. Activation and translocation of pkc- $\varepsilon$, which consequently facilitates the opening of mito- $\mathrm{K}_{\mathrm{ATP}}$ channels, have been shown to be crucial in triggering the cardioprotective effects of IPC and IPostC $[54,55,102,103]$ and were shown to be the mechanism by which diazoxide exerts its hepatic postconditioning effect [53].

NO could also underlie the protection afforded by postconditioning. In fact, it has been shown that increases in NO serum content and upregulation of NOS expression are much more prominent in IPostC treated animals; therefore, it has been postulated that e-NOS- and i-NOS-mediated NO production may be an important mechanism of this protection $[45,83]$. NO is considered a controversial mediator of physiological and pathological processes inherent in IR injury since it has been shown to have both protective and deleterious effects on cellular function [104]. In fact, several studies that have investigated the role of NO in partial liver ischemia-reperfusion models have provided controversial results [11, 104, 105]. NO is synthesized from L-arginine by three isoforms of the NOS, the e-NOS, the i-NOS, and the neuronal synthase [105]. Whether NO has a protective or deleterious effect probably depends on the source and quantity of NO produced and the cellular redox status of the liver [105-107]. On the one hand, NO stimulation under oxidative stress conditions can induce reperfusion-mediated liver injury through lipid peroxidation, DNA damage, and proapoptotic effects [105]. In the presence of superoxide, NO forms peroxynitrite, a potent oxidant agent which can decompose to generate an extremely hepatotoxic substance [108]. Therefore, apart from favorable actions, excessive production of NO may also prove deleterious and have cytotoxic potential through its interaction with superoxide anion and contribute to the hepatic injury evident in the late phases of reperfusion.

On the other hand, e-NOS-derived NO is considered to have a cytoprotective effect in IR injury, playing an important role in regulation of intracellular calcium levels 
and inhibition of platelet aggregation and counteracting the vasoconstriction caused by endothelin, particularly during the early stages of liver IR $[9,105,109]$. e-NOS expression is downregulated during liver reperfusion as a result of inhibition of e-NOS activity by oxidative stress and absence of flow within the sinusoids during ischemia [104]. The decreased production of NO from e-NOS increases the vascular resistance of the intrahepatic circulation and contributes to the microcirculatory dysfunction following reperfusion $[14,110]$. It has been shown that IR injury is exacerbated in e-NOSand i-NOS-deficient animal models $[111,112]$, whereas genetic overexpression of e-NOS has been shown to attenuate hepatic IR injury in a rat model [113]. NO production by e-NOS has also been shown to act favorably in renal and myocardial IR injury $[114,115]$. Therefore, enhanced e-NOS expression has a cytoprotective effect and acts protectively by preservation of the sinusoidal structure and maintenance of blood flow through the hepatic microcirculation [104]. Favorable effects of inhaled NO in the clinical context of liver injury have also been reported, since it was shown to accelerate restoration of liver function in adults after liver transplantation [116].

Additionally, it has already been suggested that the protective role of IPC in the liver is mediated through NO pathways $[24,117,118]$ while there is evidence implicating $\mathrm{NO}$ in the protection of IPostC in tissues other than the liver $[110,119]$. Therefore, the protective effect of IPostC in liver IR injury could similarly be related to the enhanced level of expression of e-NOS and i-NOS, which in turn increase endogenous NO production [45, 83]. Additionally, milrinone-induced postconditioning could be mediated through NOS activation, as a NOS inhibitor, injected before milrinone administration, completely abrogated the protective effects of milrinone, in the study by Toyoda et al. [52]. The postconditioning effect of Rb1 was also found to be mediated through NOS activation and increased NO production in serum and liver tissues, in the study by Guo et al. [63]. Upregulated NO by IPostC or pharmacological postconditioning might also have a role in modulating the inflammatory process by downregulating the expression of TNF- $\alpha$ and ICAM-1 $[63,83]$. In fact, NO has been reported to decrease ICAM-1 expression, which results in reduction of polymorphonucleocyte adhesion to the endothelium stimulated by TNF- $\alpha$ [120]. Additionally, NO donors have been found to attenuate leukocyte-endothelial cell reactions and adhesive interactions, thus maintaining vascular patency [121].

The exact mechanisms through which enhanced levels of NO when postconditioning is applied convey hepatoprotection are yet to be defined. Guo et al. demonstrated increased expression of HIF- $1 \alpha$ and PI3K-Akt in postconditioned animals and associated the upregulation of these two mediators with the enhanced NO generation shown in the same study [83]. NO-mediated upregulation of HIF-1 $\alpha$ was also demonstrated by postconditioning from Rb1 [63]. In fact, other studies have also shown that NO can upregulate the rate of HIF$1 \alpha$ generation $[122,123]$, while activation and upregulation of HIF- $1 \alpha$ have been found to protect liver from IR injury [124]. In turn, HIF- $1 \alpha$ has been reported to be able to improve the actions of NO [125]. Regarding PI3K-Akt, Akt is important in the activation of e-NOS-mediated NO production and NO production following Akt-mediated e-NOS activation has been shown to convey cardioprotection [126]. The PI3K-Akt pathway has also been implicated as an important signaling mediator in liver IPC $[127,128]$, while it has been shown that it plays an important role in the protective action of cardiac IPostC [129]. Therefore, the Akt/e-NOS/NO/HIF-1 $\alpha$ pathway could also play a role in the protective action of liver IPostC. It is also possible that milrinone, adenosine $A_{2} A$ receptor agonists, and Rbl-induced postconditioning could be associated with the activation of PI3K-Akt pathway and its downstream effectors, as shown in the relevant studies [52, 58,63 ]. Finally, NO could also exert its cytoprotective effects through prevention of mitochondrial permeability transition in hepatocytes through guanylyl cyclase and cyclic guanosine monophosphate-dependent kinase signaling pathway, as shown by Kim et al. [130].

The induction of HO-1 could also be implicated in the protective role of postconditioning, as shown by Zeng et al. $[46,47]$. HO-1 is upregulated by a variety of physiological and endogenous stimuli. Overexpression of HO-1 exerts cytoprotective function in a number of IR models, possibly through anti-inflammatory, antioxidant, and antiapoptotic properties, with reports of modulation of intrahepatic sinusoidal tone, improved liver microcirculation, and reduction of early oxidative burst by HO-1 and its byproducts [131133]. HO-1 also potentiates the survival of small-for-size liver grafts [134], while some studies have suggested that IPC may exert its protective effects and suppress systemic inflammatory responses via enhanced HO-1 expression [135138]. Additionally, it has been shown that lung IPostC attenuates lung ischemic reperfusion injury through HO-1 upregulation $[139,140]$. Therefore, it could also be possible that the protective effects of hepatic postconditioning could also be mediated by upregulation of $\mathrm{HO}-1$ expression $[46,47]$.

\section{Conclusion}

Despite the favorable effects of IPC, documented in a variety of experimental and some clinical settings, it has an obvious disadvantage: it must be initiated before the ischemic event, which is not always a feasible option. In contrast, the protection afforded by IPostC, which could be seen as an attempt for slow intermittent oxygenation through several cycles of on/off flow before permanent reperfusion, could be considered as a more appropriate choice with a theoretical clinical application value. Especially in the context of deceased donor liver transplantation, the onset of ischemia cannot be predicted and therefore postconditioning is a more appealing strategy for clinical implementation, since the onset of reperfusion is more predictable and manipulations targeting this period can be more attractive. Even in the setting of hepatectomies, preconditioning requires timely planning and is not suitable for emergency situations. However, intermittent interruptions of blood flow in the early phase of reperfusion seem to be a more suitable alternative, since the onset of reperfusion is easy to define and can be applied selectively with precisely controlled timing in 
patients in need for unpredictably prolonged periods of inflow occlusion.

A growing body of evidence shows that IPC and IPostC share many similarities in the mechanisms involved. However, unlike IPC, IPostC focuses on the early events of reperfusion and applies the brief episodes of ischemia at the onset of revascularization, basically constituting a variation of controlled reperfusion. It could be considered as a process that targets the first few minutes of reperfusion that modifies reperfusion injury rendering the cell and mitochondria more tolerant to the biochemical and metabolic perturbation that occurs in the transition from ischemia to reperfusion. Moreover, it has been postulated that when ischemic tissues are reperfused straight away, abrupt reperfusion flow washes out endogenous protective substances, while a slower and more controlled reperfusion with the short series of repetitive cycles of reperfusion and reocclusion maintains protective substances inside the liver parenchyma for longer.

The beneficial effects of IPostC have been documented in several experimental studies in different organs [141] and confirmed in human clinical studies in the heart [34, 35]. As explored in the current review, there have been several experimental studies of the benefits of IPostC for the liver but we were able to identify just one very recent study of its use in the clinical setting, in the context of adult cadaveric liver transplantation [48]. Unlike donor preconditioning, which is not always feasible as mentioned above, graft postconditioning in the recipient seems to be a more appealing strategy since it can be applied selectively in settings prone to greater risks for IR injury due to donor-, procurement-, or recipientrelated factors and can prove useful in complex cases requiring long periods of ischemia or with marginal grafts. Based on these findings, it seems reasonable to explore the possibility of the use of IPostC in the context of clinical liver surgery beyond transplantation, especially in complex cases needing long periods of ischemia, in cases of unexpected ischemia, where there is limited clinical applicability for IPC, and during major hepatectomies, with marginal liver remnants. In the light of this, a $30 \%$ reduction of liver cell necrosis was demonstrated with the application of IPostC by Knudsen et al. [43]. If this is extrapolated to the clinical context, a necrosis reduction of this size could be crucial for patients subjected to large liver resections. Such resections can be complicated by bleeding, which is often unpredictable, and bleeding has to be controlled by clamping. In this context, IPC is not a feasible option and IPostC is the technique of choice because it can be applied after clamping to ensure the survival of as much liver parenchyma as possible. Apart from the reduction of liver cell necrosis, the favorable effect of IPostC on liver regeneration $[71,74,75]$ as well as the requirement for less operative time in contrast to preconditioning techniques makes postconditioning clinically more attractive to implement in the context of extended hepatectomies.

It has been shown that the early period of reperfusion is an important period for salvaging ischemic tissue since rapid production of ROS, opening of $\mathrm{MPTP}$, and fluctuations in $\mathrm{pH}$ develop during this period [43, 98, 101]. Moreover, results from Zhao et al. in cardiac tissues have demonstrated that manipulation of the early reperfusion phase is crucial for the protective effect of IPostC [31]. In fact, when the maneuver of IPostC was delayed for a while, instead of being applied at the very onset of reperfusion, its protective effect was lost [32]. Therefore, proper choice of timing and number of reperfusion/reocclusion cycles should be refined in future studies in the setting of liver surgery. Pharmacological inducers of postconditioning could also be useful in alleviating the metabolic, structural, and functional changes of IR injury and should be thoroughly evaluated. Future studies will be required to standardize the postconditioning procedure, to clarify its clinical impact, and to deepen its molecular understanding.

\section{Abbreviations}

\begin{tabular}{|c|c|}
\hline IR: & Ischemia-reperfusion \\
\hline ROS: & Reactive oxygen species \\
\hline NO: & Nitric oxide \\
\hline IPC: & Ischemic preconditioning \\
\hline IPostC: & Ischemic postconditioning \\
\hline MDA: & Malondialdehyde \\
\hline SOD: & Superoxide dismutase \\
\hline CAT: & Catalase \\
\hline GSH-P ${ }_{X}$ : & Glutathione peroxidase \\
\hline TUNEL: & $\begin{array}{l}\text { Terminal deoxynucleotidyl transferase } \\
\text { dUTP nick end labeling }\end{array}$ \\
\hline ALT: & Alanine aminotransferase \\
\hline AST: & Aspartate aminotransferase \\
\hline$N F-\kappa B:$ & Nuclear factor-kappa beta \\
\hline 4-HNE: & 4-Hydroxy-2-nonenal \\
\hline NVR: & Necrotic volume ratio \\
\hline LDH: & Lactate dehydrogenase \\
\hline MPO: & Myeloperoxidase \\
\hline RT-PCR: & $\begin{array}{l}\text { Reverse transcriptase-polymerase chain } \\
\text { reaction }\end{array}$ \\
\hline TNF- $\alpha$ : & Tumor necrosis factor-alpha \\
\hline MIP-2: & Macrophage inflammatory protein-2 \\
\hline i-NOS: & Inducible NO synthase \\
\hline e-NOS: & Endothelial NO synthase \\
\hline HO-1: & Heme oxygenase- 1 \\
\hline cAMP: & Cyclic adenosine monophosphate \\
\hline PKA: & Protein kinase $\mathrm{A}$ \\
\hline PI3K-Akt: & Phosphatidylinositol 3-kinase \\
\hline mito-K $\mathrm{K}_{\mathrm{ATP}}$ : & ATP-dependent mitochondrial potassium \\
\hline pkc- $\varepsilon:$ & Protein kinase c-epsilon \\
\hline Rb1: & Ginsenoside Rb1 \\
\hline rhEPO: & Human recombinant erythropoietin \\
\hline HIF-1 $\alpha$ : & Hypoxia inducible factor 1-alpha \\
\hline VEGF: & Vascular endothelial growth factor \\
\hline ICAM-1: & Intercellular adhesion molecule-1. \\
\hline
\end{tabular}

\section{Competing Interests}

The authors declare that they have no competing interests.

\section{References}

[1] M. T. de Boer, I. Q. Molenaar, and R. J. Porte, "Impact of blood loss on outcome after liver resection," Digestive Surgery, vol. 24, no. 4, pp. 259-264, 2007. 
[2] N. N. Rahbari, M. N. Wente, P. Schemmer et al., "Systematic review and meta-analysis of the effect of portal triad clamping on outcome after hepatic resection," British Journal of Surgery, vol. 95, no. 4, pp. 424-432, 2008.

[3] J. D. W. van der Bilt, D. P. Livestro, A. Borren, R. Van Hillegersberg, and I. H. M. Borel Rinkes, "European survey on the application of vascular clamping in liver surgery," Digestive Surgery, vol. 24, no. 6, pp. 423-432, 2008.

[4] Y.-I. Kim, "Ischemia-reperfusion injury of the human liver during hepatic resection," Journal of Hepato-Biliary-Pancreatic Surgery, vol. 10, no. 3, pp. 195-199, 2003.

[5] F. Serracino-Inglott, N. A. Habib, and R. T. Mathie, "Hepatic ischemia-reperfusion injury," American Journal of Surgery, vol. 181, no. 2, pp. 160-166, 2001.

[6] Y. Zhai, H. Petrowsky, J. C. Hong, R. W. Busuttil, and J. W. Kupiec-Weglinski, "Ischaemia-reperfusion injury in liver transplantation-from bench to bedside," Nature Reviews Gastroenterology and Hepatology, vol. 10, no. 2, pp. 79-89, 2013.

[7] D. Papadopoulos, T. Siempis, E. Theodorakou, and G. Tsoulfas, "Hepatic ischemia and reperfusion injury and trauma: current concepts," Archives of Trauma Research, vol. 2, no. 2, pp. 63-70, 2013.

[8] A. J. Vardanian, R. W. Busuttil, and J. W. Kupiec-Weglinski, "Molecular mediators of liver ischemia and reperfusion injury: a brief review," Molecular Medicine, vol. 14, no. 5-6, pp. 337-345, 2008.

[9] G. Datta, B. J. Fuller, and B. R. Davidson, "Molecular mechanisms of liver ischemia reperfusion injury: insights from transgenic knockout models," World Journal of Gastroenterology, vol. 19, no. 11, pp. 1683-1698, 2013.

[10] M. Elias-Miró, M. B. Jiménez-Castro, J. Rodés, and C. Peralta, "Current knowledge on oxidative stress in hepatic ischemia/reperfusion," Free Radical Research, vol. 47, no. 8, pp. 555-568, 2013.

[11] A. Siriussawakul, A. Zaky, and J. D. Lang, "Role of nitric oxide in hepatic ischemia-reperfusion injury," World Journal of Gastroenterology, vol. 16, no. 48, pp. 6079-6086, 2010.

[12] H. Jaeschke, "Reactive oxygen and mechanisms of inflammatory liver injury: present concepts," Journal of Gastroenterology and Hepatology, vol. 26, supplement 1, pp. 173-179, 2011.

[13] E. E. Montalvo-Jave, T. Escalante-Tattersfield, J. A. OrtegaSalgado, E. Piña, and D. A. Geller, "Factors in the pathophysiology of the liver ischemia-reperfusion injury," Journal of Surgical Research, vol. 147, no. 1, pp. 153-159, 2008.

[14] M. D. Menger, S. Richter, J. Yamauchi, and B. Vollmar, "Role of microcirculation in hepatic ischemia/reperfusion injury," Hepato-Gastroenterology, vol. 46, supplement 2, pp. 1452-1457, 1999.

[15] C. Peralta, M. B. Jiménez-Castro, and J. Gracia-Sancho, "Hepatic ischemia and reperfusion injury: effects on the liver sinusoidal milieu," Journal of Hepatology, vol. 59, no. 5, pp. 10941106, 2013.

[16] H. Malhi, G. J. Gores, and J. J. Lemasters, "Apoptosis and necrosis in the liver: a tale of two deaths?" Hepatology, vol. 43, no. 2, pp. S31-S44, 2006.

[17] H. A. Rüdiger, R. Graf, and P.-A. Clavien, "Liver ischemia: apoptosis as a central mechanism of injury," Journal of Investigative Surgery, vol. 16, no. 3, pp. 149-159, 2003.

[18] C. Nastos, K. Kalimeris, N. Papoutsidakis et al., "Global consequences of liver ischemia/reperfusion injury," Oxidative Medicine and Cellular Longevity, vol. 2014, Article ID 906965 , 13 pages, 2014.
[19] C. E. Murry, R. B. Jennings, and K. A. Reimer, "Preconditioning with ischemia: a delay of lethal cell injury in ischemic myocardium," Circulation, vol. 74, no. 5, pp. 1124-1136, 1986.

[20] K. S. Gurusamy, H. D. Gonzalez, and B. R. Davidson, "Current protective strategies in liver surgery," World Journal of Gastroenterology, vol. 16, no. 48, pp. 6098-6103, 2010.

[21] K. Theodoraki, A. Tympa, I. Karmaniolou, A. Tsaroucha, N. Arkadopoulos, and V. Smyrniotis, "Ischemia/reperfusion injury in liver resection: a review of preconditioning methods," Surgery Today, vol. 41, no. 5, pp. 620-629, 2011.

[22] K. K. Desai, G. S. Dikdan, A. Shareef, and B. Koneru, "Ischemic preconditioning of the liver: a few perspectives from the bench to bedside translation," Liver Transplantation, vol. 14, no. 11, pp. 1569-1577, 2008.

[23] S. Suzuki, K. Inaba, and H. Konno, "Ischemic preconditioning in hepatic ischemia and reperfusion," Current Opinion in Organ Transplantation, vol. 13, no. 2, pp. 142-147, 2008.

[24] E. Alchera, C. Dal Ponte, C. Imarisio, E. Albano, and R. Carini, "Molecular mechanisms of liver preconditioning," World Journal of Gastroenterology, vol. 16, no. 48, pp. 6058-6067, 2010.

[25] N. Arkadopoulos, G. Kostopanagiotou, K. Theodoraki et al., "Ischemic preconditioning confers antiapoptotic protection during major hepatectomies performed under combined inflow and outflow exclusion of the liver. A randomized clinical trial," World Journal of Surgery, vol. 33, no. 9, pp. 1909-1915, 2009.

[26] M. Glanemann, B. Vollmar, A. K. Nussler, T. Schaefer, P. Neuhaus, and M. D. Menger, "Ischemic preconditioning protects from hepatic ischemia/reperfusion-injury by preservation of microcirculation and mitochondrial redox-state," Journal of Hepatology, vol. 38, no. 1, pp. 59-66, 2003.

[27] K. Theodoraki, N. Arkadopoulos, G. Fragulidis et al., "Ischemic preconditioning attenuates lactate release by the liver during hepatectomies under vascular control: a case-control study," Journal of Gastrointestinal Surgery, vol. 15, no. 4, pp. 589-597, 2011.

[28] K. S. Gurusamy, Y. Kumar, V. Pamecha, D. Sharma, and B. R. Davidson, "Ischaemic pre-conditioning for elective liver resections performed under vascular occlusion," Cochrane Database of Systematic Reviews, no. 1, Article ID CD007629, 2009.

[29] S. O’Neill, S. Leuschner, S. J. McNally, O. J. Garden, S. J. Wigmore, and E. M. Harrison, "Meta-analysis of ischaemic preconditioning for liver resections," British Journal of Surgery, vol. 100, no. 13, pp. 1689-1700, 2013.

[30] P. N. A. Martins, "Clinical translation of remote ischemic preconditioning of the liver," Annals of Surgery, vol. 254, no. 1, p. 180, 2011.

[31] Z.-Q. Zhao, J. S. Corvera, M. E. Halkos et al., "Inhibition of myocardial injury by ischemic postconditioning during reperfusion: comparison with ischemic preconditioning," American Journal of Physiology-Heart and Circulatory Physiology, vol. 285, pp. H579-H588, 2003.

[32] H. Kin, Z.-Q. Zhao, H.-Y. Sun et al., "Postconditioning attenuates myocardial ischemia-reperfusion injury by inhibiting events in the early minutes of reperfusion," Cardiovascular Research, vol. 62, no. 1, pp. 74-85, 2004.

[33] R. A. Kloner, J. Dow, and A. Bhandari, "Postconditioning markedly attenuates ventricular arrhythmias after ischemiareperfusion," Journal of Cardiovascular Pharmacology and Therapeutics, vol. 11, no. 1, pp. 55-63, 2006.

[34] P. Staat, G. Rioufol, C. Piot et al., "Postconditioning the human heart," Circulation, vol. 112, no. 14, pp. 2143-2148, 2005. 
[35] H. Thibault, C. Piot, P. Staat et al., "Long-term benefit of postconditioning," Circulation, vol. 117, no. 8, pp. 1037-1044, 2008.

[36] P. R. Hansen, H. Thibault, and J. Abdulla, "Postconditioning during primary percutaneous coronary intervention: a review and meta-analysis," International Journal of Cardiology, vol. 144, no. 1, pp. 22-25, 2010.

[37] K. Sun, Z.-S. Liu, and Q. Sun, "Role of mitochondria in cell apoptosis during hepatic ischemia-reperfusion injury and protective effect of ischemic postconditioning," World Journal of Gastroenterology, vol. 10, no. 13, pp. 1934-1938, 2004.

[38] W.-X. Zhang, W. Yin, L. Zhang et al., "Preconditioning and postconditioning reduce hepatic ischemia-reperfusion injury in rats," Hepatobiliary and Pancreatic Diseases International, vol. 8, no. 6, pp. 586-590, 2009.

[39] S.-Y. Yoon, C. Y. Kim, H. J. Han, K. O. Lee, and T.-J. Song, "Protective effect of ischemic postconditioning against hepatic ischemic reperfusion injury in rat liver," Annals of Surgical Treatment and Research, vol. 88, no. 5, pp. 241-245, 2015.

[40] A. R. F. Teixeira, N. T. Molan, M. S. Kubrusly et al., "Postconditioning ameliorates lipid peroxidation in liver ischemiareperfusion injury in rats," Acta Cirurgica Brasileira, vol. 24, no. 1, pp. 52-56, 2009.

[41] C. H. M. Dos Santos, J. C. D. V. Pontes, L. N. O. Miiji, D. I. Nakamura, C. A. V. Galhardo, and S. M. Aguena, "Postconditioning effect in the hepatic ischemia and reperfusion in rats," Acta Cirurgica Brasileira, vol. 25, no. 2, pp. 163-168, 2010.

[42] H.-C. Lin, T.-K. Lee, C.-C. Tsai, I.-R. Lai, and K.-S. Lu, "Ischemic postconditioning protects liver from ischemiareperfusion injury by modulating mitochondrial permeability transition," Transplantation, vol. 93, no. 3, pp. 265-271, 2012.

[43] A. R. Knudsen, A.-S. Kannerup, H. Grønbæk et al., "Quantitative histological assessment of hepatic ischemia-reperfusion injuries following ischemic pre- and post-conditioning in the rat liver," Journal of Surgical Research, vol. 180, no. 1, pp. ell-e20, 2013.

[44] S. M. Niehues, J. K. Unger, M. Malinowski, J. Neymeyer, B. Hamm, and M. Stockmann, "Liver volume measurement: reason of the difference between in vivo CT-volumetry and intraoperative ex vivo determination and how to cope it," European Journal of Medical Research, vol. 15, no. 8, pp. 345350,2010 .

[45] N. Wang, J. G. Lu, X. L. He et al., "Effects of ischemic postconditioning on reperfusion injury in rat liver grafts after orthotopic liver transplantation," Hepatology Research, vol. 39, no. 4, pp. 382-390, 2009.

[46] Z. Zeng, H. F. Huang, M. Q. Chen, F. Song, and Y. J. Zhang, "Postconditioning prevents ischemia/reperfusion injury in rat liver transplantation," Hepatogastroenterology, vol. 57, no. 101, pp. 875-881, 2010.

[47] Z. Zeng, H. F. Huang, M. Q. Chen, F. Song, and Y. J. Zhang, "Contributions of heme oxygenase-1 in postconditioningprotected ischemia-reperfusion injury in rat liver transplantation," Transplantation Proceedings, vol. 43, no. 7, pp. 2517-2523, 2011.

[48] L. Ricca, A. Lemoine, F. Cauchy et al., "Ischemic postconditioning of the liver graft in adult liver transplantation," Transplantation, vol. 99, no. 8, pp. 1633-1643, 2015.

[49] T. Minor, J. Stegemann, A. Hirner, and M. Koetting, "Impaired autophagic clearance after cold preservation of fatty livers correlates with tissue necrosis upon reperfusion and is reversed by hypothermic reconditioning," Liver Transplantation, vol. 15, no. 7, pp. 798-805, 2009.

[50] A. Lanir, R. L. Jenkins, C. Caldwell, R. G. L. Lee, U. Khettry, and M. E. Clouse, "Hepatic transplantation survival: correlation with adenine nucleotide level in donor liver," Hepatology, vol. 8, no. 3, pp. 471-475, 1988.

[51] K. Satoh, M. Kume, Y. Abe et al., "Implication of protein kinase A for a hepato-protective mechanism of milrinone pretreatment," Journal of Surgical Research, vol. 155, no. 1, pp. 32-39, 2009.

[52] T. Toyoda, S. Tosaka, R. Tosaka et al., "Milrinone-induced postconditioning reduces hepatic ischemia-reperfusion injury in rats: the roles of phosphatidylinositol 3-kinase and nitric oxide," Journal of Surgical Research, vol. 186, no. 1, pp. 446-451, 2014.

[53] Y. S. Tian, T. Z. Rong, Y. L. Hong, L. Min, and P. G. Jian, "Pharmacological postconditioning with diazoxide attenuates ischemia/reperfusion-induced injury in rat liver," Experimental and Therapeutic Medicine, vol. 5, no. 4, pp. 1169-1173, 2013.

[54] G. S. Liu, M. V. Cohen, D. Mochly-Rosen, and J. M. Downey, "Protein kinase C- $\varepsilon$ is responsible for the protection of preconditioning in rabbit cardiomyocytes," Journal of Molecular and Cellular Cardiology, vol. 31, no. 10, pp. 1937-1948, 1999.

[55] H. Liu, H. Y. Zhang, X. Zhu, Z. Shao, and Z. Yao, "Preconditioning blocks cardiocyte apoptosis: role of K(ATP) channels and PKC-epsilon," American Journal of Physiology-Heart and Circulatory Physiology, vol. 282, no. 4, pp. H1380-H1386, 2002.

[56] B. Beck-Schimmer, S. Breitenstein, J. M. Bonvini et al., "Protection of pharmacological postconditioning in liver surgery: results of a prospective randomized controlled trial," Annals of Surgery, vol. 256, no. 5, pp. 837-845, 2012.

[57] B. Beck-Schimmer, S. Breitenstein, S. Urech et al., "A randomized controlled trial on pharmacological preconditioning in liver surgery using a volatile anesthetic," Annals of Surgery, vol. 248, no. 6, pp. 909-918, 2008.

[58] C. Dal Ponte, E. Alchera, A. Follenzi et al., "Pharmacological postconditioning protects against hepatic ischemia/reperfusion injury," Liver Transplantation, vol. 17, no. 4, pp. 474-482, 2011.

[59] H. Nakayama, Y. Yamamoto, M. Kume et al., "Pharmacologic stimulation of adenosine A2 receptor supplants ischemic preconditioning in providing ischemic tolerance in rat livers," Surgery, vol. 126, no. 5, pp. 945-954, 1999.

[60] C. Peralta, G. Hotter, D. Closa et al., "The protective role of adenosine in inducing nitric oxide synthesis in rat liver ischemia preconditioning is mediated by activation of adenosine A2 receptors," Hepatology, vol. 29, no. 1, pp. 126-132, 1999.

[61] J. Wang, L. Qiao, Y. Li, and G. Yang, "Ginsenoside Rb1 attenuates intestinal ischemia-reperfusion-induced liver injury by inhibiting NF- $\kappa \mathrm{B}$ activation," Experimental and Molecular Medicine, vol. 40, no. 6, pp. 686-698, 2008.

[62] H.-U. Lee, E.-A. Bae, M. J. Han, N.-J. Kim, and D.-H. Kim, "Hepatoprotective effect of ginsenoside Rbl and compound $\mathrm{K}$ on tert-butyl hydroperoxide-induced liver injury," Liver International, vol. 25, no. 5, pp. 1069-1073, 2005.

[63] Y. Guo, T. Yang, J. Lu et al., "Rb1 postconditioning attenuates liver warm ischemia-reperfusion injury through ROS-NO-HIF pathway," Life Sciences, vol. 88, no. 13-14, pp. 598-605, 2011.

[64] H. M. Shawky, S. M. Younan, L. A. Rashed, and H. Shoukry, "Effect of recombinant erythropoietin on ischemiareperfusion-induced apoptosis in rat liver," Journal of Physiology and Biochemistry, vol. 68, no. 1, pp. 19-28, 2012. 
[65] B.-Q. Wu, W.-W. Chu, L.-Y. Zhang, P. Wang, Q.-Y. Ma, and D.$\mathrm{H}$. Wang, "Protection of preconditioning, postconditioning and combined therapy against hepatic ishemia/reperfusion injury," Chinese Journal of Traumatology - English Edition, vol. 10, no. 4, pp. 223-227, 2007.

[66] K. X. Wang, S. Y. Hu, X. S. Jiang et al., "Protective effects of ischaemic postconditioning on warm/cold ischaemic reperfusion injury in rat liver: a comparative study with ischaemic preconditioning," Chinese Medical Journal, vol. 121, no. 20, pp. 2004-2009, 2008.

[67] X. Song, N. Zhang, H. Hu, L. Cao, and H. Zhang, "Combined preconditioning and postconditioning provides synergistic protection against liver ischemic reperfusion injury," International Journal of Biological Sciences, vol. 8, no. 5, pp. 707-718, 2012.

[68] M. L. Lemus-Varela, M. E. Flores-Soto, R. Cervantes-Munguía et al., "Expression of HIF-1 $\alpha$, VEGF and EPO in peripheral blood from patients with two cardiac abnormalities associated with hypoxia," Clinical Biochemistry, vol. 43, no. 3, pp. 234-239, 2010.

[69] M. Bockhorn, M. Goralski, D. Prokofiev et al., "VEGF is important for early liver regeneration after partial hepatectomy," Journal of Surgical Research, vol. 138, no. 2, pp. 291-299, 2007.

[70] A. R. Knudsen, A.-S. Kannerup, H. Grønbæk et al., "Effects of ischemic pre- and postconditioning on HIF- $1 \alpha$, VEGF and TGF- $\beta$ expression after warm ischemia and reperfusion in the rat liver," Comparative Hepatology, vol. 10, article 3, 2011.

[71] A. R. Knudsen, A.-S. Kannerup, R. Dich et al., "Expression of genes involved in rat liver angiogenesis after ischaemia and reperfusion: effects of ischaemic pre- and post-conditioning," $H P B$, vol. 12, no. 8, pp. 554-560, 2010.

[72] I. B. Nicoud, C. M. Jones, J. M. Pierce et al., "Warm hepatic ischemia-reperfusion promotes growth of colorectal carcinoma micrometastases in mouse liver via matrix metalloproteinase-9 induction," Cancer Research, vol. 67, no. 6, pp. 2720-2728, 2007.

[73] J. D. W. van der Bilt, O. Kranenburg, M. W. Nijkamp et al., "Ischemia/reperfusion accelerates the outgrowth of hepatic micrometastases in a highly standardized murine model," Hepatology, vol. 42, no. 1, pp. 165-175, 2005.

[74] A. R. Knudsen, A.-S. Kannerup, R. Dich et al., "Ischemic pre- and postconditioning has pronounced effects on gene expression profiles in the rat liver after ischemia/reperfusion," American Journal of Physiology-Gastrointestinal and Liver Physiology, vol. 303, no. 4, pp. G482-G489, 2012.

[75] S. B. Young, A. R. C. Pires, G. T. Boaventura, A. M. R. Ferreira, J. M. S. G. Martinho, and M. A. Galhardo, "Effect of ischemic preconditioning and postconditioning on liver regeneration in prepubertal rats," Transplantation Proceedings, vol. 46, no. 6, pp. 1867-1871, 2014.

[76] M. Kerem, A. Bedirli, E. Ofluoglu et al., "Ischemic preconditioning improves liver regeneration by sustaining energy metabolism after partial hepatectomy under ischemia in rats," Liver International, vol. 26, no. 8, pp. 994-999, 2006.

[77] A. Bedirli, M. Kerem, H. Pasaoglu, O. Erdem, E. Ofluoglu, and O. Sakrak, "Effects of ischemic preconditioning on regenerative capacity of hepatocyte in the ischemically damaged rat livers," Journal of Surgical Research, vol. 125, no. 1, pp. 42-48, 2005.

[78] D. Del Rio, A. J. Stewart, and N. Pellegrini, "A review of recent studies on malondialdehyde as toxic molecule and biological marker of oxidative stress," Nutrition, Metabolism and Cardiovascular Diseases, vol. 15, no. 4, pp. 316-328, 2005.

[79] Y. M. Tsutsumi, T. Yokoyama, Y. Horikawa, D. M. Roth, and H. H. Patel, "Reactive oxygen species trigger ischemic and pharmacological postconditioning: in vivo and in vitro characterization," Life Sciences, vol. 81, no. 15, pp. 1223-1227, 2007.

[80] C. Penna, D. Mancardi, R. Rastaldo, and P. Pagliaro, "Cardioprotection: a radical view. Free radicals in pre and postconditioning," Biochimica et Biophysica Acta-Bioenergetics, vol. 1787, no. 7, pp. 781-793, 2009.

[81] T. C. Nichols, "NF- $\kappa$ B and reperfusion injury," Drug News and Perspectives, vol. 17, no. 2, pp. 99-104, 2004.

[82] H. Kin, N.-P. Wang, J. Mykytenko et al., "Inhibition of myocardial apoptosis by postconditioning is associated with attenuation of oxidative stress-mediated nuclear factor- $\kappa \mathrm{B}$ translocation and TNF $\alpha$ release," Shock, vol. 29, no. 6, pp. 761-768, 2008.

[83] J. Y. Guo, T. Yang, X. G. Sun et al., "Ischemic postconditioning attenuates liver warm ischemia-reperfusion injury through Akt-eNOS-NO-HIF pathway," Journal of Biomedical Science, vol. 18, article 79, 2011.

[84] S. S. Yadav, D. N. Howell, W. Gao, D. A. Steeber, R. C. Harland, and P.-A. Clavien, "L-selectin and ICAM-1 mediate reperfusion injury and neutrophil adhesion in the warm ischemic mouse liver," American Journal of Physiology-Gastrointestinal and Liver Physiology, vol. 275, no. 6, pp. G1341-G1352, 1998.

[85] S. Marubayashi, Y. Oshiro, T. Maeda et al., "Protective effect of monoclonal antibodies to adhesion molecules on rat liver ischemia-reperfusion injury," Surgery, vol. 122, no. 1, pp. 45-52, 1997.

[86] H. Nakano, M. Kuzume, K. Namatame, M. Yamaguchi, and K. Kumada, "Efficacy of intraportal injection of anti-ICAM-1 monoclonal antibody against liver cell injury following warm ischemia in the rat," The American Journal of Surgery, vol. 170, no. 1, pp. 64-66, 1995.

[87] H. Jaeschke, A. P. Bautista, Z. Spolarics, and J. J. Spitzer, "Superoxide generation by neutrophils and Kupffer cells during in vivo reperfusion after hepatic ischemia in rats," Journal of Leukocyte Biology, vol. 52, no. 4, pp. 377-382, 1992.

[88] P. R. Hansen, "Role of neutrophils in myocardial ischemia and reperfusion," Circulation, vol. 91, no. 6, pp. 1872-1885, 1995.

[89] K. Yamanouchi, S. Eguchi, Y. Kamohara et al., "Glycine reduces hepatic warm ischaemia-reperfusion injury by suppressing inflammatory reactions in rats," Liver International, vol. 27, no. 9, pp. 1249-1254, 2007.

[90] M. Kataoka, H. Shimizu, N. Mitsuhashi et al., "Effect of coldischemia time on C-X-C chemokine expression and neutrophil accumulation in the graft liver after orthotopic liver transplantation in rats," Transplantation, vol. 73, no. 11, pp. 1730-1735, 2002.

[91] F. Di Lisa, M. Canton, R. Menabò, G. Dodoni, and P. Bernardi, "Mitochondria and reperfusion injury. The role of permeability transition," Basic Research in Cardiology, vol. 98, no. 4, pp. 235241, 2003.

[92] T. Wakabayashi, "Structural changes of mitochondria related to apoptosis: swelling and megamitochondria formation," Acta Biochimica Polonica, vol. 46, no. 2, pp. 223-237, 1999.

[93] E. Finkel, "Cell biology: the mitochondrion: is it central to apoptosis?” Science, vol. 292, no. 5517, pp. 624-626, 2001.

[94] M. Crompton, "Mitochondrial intermembrane junctional complexes and their role in cell death," The Journal of Physiology, vol. 529, no. 1, pp. 11-21, 2000.

[95] P. Marchetti, M. Castedo, S. A. Susin et al., "Mitochondrial permeability transition is a central coordinating event of apoptosis," The Journal of Experimental Medicine, vol. 184, no. 3, pp. 1155-1160, 1996. 
[96] V. P. Skulachev, "Cytochrome $\mathrm{c}$ in the apoptotic and antioxidant cascades," FEBS Letters, vol. 423, no. 3, pp. 275-280, 1998.

[97] T. P. Theruvath, Z. Zhong, P. Pediaditakis et al., "Minocycline and N-methyl-4-isoleucine cyclosporin (NIM811) mitigate storage/reperfusion injury after rat liver transplantation through suppression of the mitochondrial permeability transition," Hepatology, vol. 47, no. 1, pp. 236-246, 2008.

[98] O. Gateau-Roesch, L. Argaud, and M. Ovize, "Mitochondrial permeability transition pore and postconditioning," Cardiovascular Research, vol. 70, no. 2, pp. 264-273, 2006.

[99] L. Argaud, O. Gateau-Roesch, O. Raisky, J. Loufouat, D. Robert, and M. Ovize, "Postconditioning inhibits mitochondrial permeability transition," Circulation, vol. 111, no. 2, pp. 194-197, 2005.

[100] S. Y. Lim, S. M. Davidson, D. J. Hausenloy, and D. M. Yellon, "Preconditioning and postconditioning: the essential role of the mitochondrial permeability transition pore," Cardiovascular Research, vol. 75, no. 3, pp. 530-535, 2007.

[101] M. V. Cohen, X.-M. Yang, and J. M. Downey, “The pH hypothesis of postconditioning: staccato reperfusion reintroduces oxygen and perpetuates myocardial acidosis," Circulation, vol. 115, no. 14, pp. 1895-1903, 2007.

[102] A. J. Zatta, H. Kin, G. Lee et al., "Infarct-sparing effect of myocardial postconditioning is dependent on protein kinase $\mathrm{C}$ signalling," Cardiovascular Research, vol. 70, no. 2, pp. 315-324, 2006.

[103] C. Jin, J. Wu, M. Watanabe, T. Okada, and T. Iesaki, "Mitochondrial $\mathrm{K}^{+}$channels are involved in ischemic postconditioning in rat hearts," Journal of Physiological Sciences, vol. 62, no. 4, pp. 325-332, 2012.

[104] F. Serracino-Inglott, I. T. Virlos, N. A. Habib, R. C. N. Williamson, and R. T. Mathie, "Differential nitric oxide synthase expression during hepaticischemia-reperfusion," American Journal of Surgery, vol. 185, no. 6, pp. 589-595, 2003.

[105] M. Abu-Amara, S. Y. Yang, A. Seifalian, B. Davidson, and B. Fuller, "The nitric oxide pathway-evidence and mechanisms for protection against liver ischaemia reperfusion injury," Liver International, vol. 32, no. 4, pp. 531-543, 2012.

[106] D. L. Diesen and P. C. Kuo, "Nitric oxide and redox regulation in the liver: part II. Redox biology in pathologic hepatocytes and implications for intervention," Journal of Surgical Research, vol. 167, no. 1, pp. 96-112, 2011.

[107] T. Chen, R. Zamora, B. Zuckerbraun, and T. R. Billiar, "Role of nitric oxide in liver injury," Current Molecular Medicine, vol. 3, no. 6, pp. 519-526, 2003.

[108] J. S. Beckman, T. W. Beckman, J. Chen, P. A. Marshall, and B. A. Freeman, "Apparent hydroxyl radical production by peroxynitrite: implications for endothelial injury from nitric oxide and superoxide," Proceedings of the National Academy of Sciences of the United States of America, vol. 87, no. 4, pp. 1620$1624,1990$.

[109] C.-H. Cottart, L. Do, M.-C. Blanc et al., "Hepatoprotective effect of endogenous nitric oxide during ischemia-reperfusion in the rat," Hepatology, vol. 29, no. 3, pp. 809-813, 1999.

[110] B. Vollmar, J. Glasz, R. Leiderer, S. Post, and M. D. Menger, "Hepatic microcirculatory perfusion failure is a determinant of liver dysfunction in warm ischemia-reperfusion," American Journal of Pathology, vol. 145, no. 6, pp. 1421-1431, 1994.

[111] Y. Abe, I. N. Hines, G. Zibari et al., "Mouse model of liver ischemia and reperfusion injury: method for studying reactive oxygen and nitrogen metabolites in vivo," Free Radical Biology and Medicine, vol. 46, no. 1, pp. 1-7, 2009.
[112] I. N. Hines, H. Harada, S. Bharwani, K. P. Pavlick, J. M. Hoffman, and M. B. Grisham, "Enhanced post-ischemic liver injury in iNOS-deficient mice: a cautionary note," Biochemical and Biophysical Research Communications, vol. 284, no. 4, pp. 972-976, 2001.

[113] M. R. Duranski, J. W. Elrod, J. W. Calvert, N. S. Bryan, M. Feelisch, and D. J. Lefer, "Genetic overexpression of eNOS attenuates hepatic ischemia-reperfusion injury," American Journal of Physiology-Heart and Circulatory Physiology, vol. 291, no. 6, pp. H2980-H2986, 2006.

[114] A. B. Milsom, N. S. A. Patel, E. Mazzon et al., "Role for endothelial nitric oxide synthase in nitrite-induced protection against renal ischemia-reperfusion injury in mice," Nitric Oxide-Biology and Chemistry, vol. 22, no. 2, pp. 141-148, 2010.

[115] S. P. Jones, J. J. M. Greer, A. K. Kakkar et al., "Endothelial nitric oxide synthase overexpression attenuates myocardial reperfusion injury," American Journal of Physiology-Heart and Circulatory Physiology, vol. 286, no. 1, pp. H276-H282, 2004.

[116] J. D. Lang Jr., X. Teng, P. Chumley et al., "Inhaled NO accelerates restoration of liver function in adults following orthotopic liver transplantation," The Journal of Clinical Investigation, vol. 117, no. 9, pp. 2583-2591, 2007.

[117] Q. Xue, Z. Yuan, Z. Chen, R. Hao, C. Liu, and B. Tu, "Protective role of nitric oxide induced by ischemic preconditioning on cold ischemic-reperfusion injury of rat liver graft," Transplantation Proceedings, vol. 44, no. 4, pp. 948-951, 2012.

[118] C. Peralta, G. Hotter, D. Closa, E. Gelpí, O. Bulbena, and J. Roselló-Catafau, "Protective effect of preconditioning on the injury associated to hepatic ischemia-reperfusion in the rat: role of nitric oxide and adenosine," Hepatology, vol. 25, no. 4, pp. 934-937, 1997.

[119] P. Gulati and N. Singh, "Pharmacological evidence for connection of nitric oxide-mediated pathways in neuroprotective mechanism of ischemic postconditioning in mice," Journal of Pharmacy and Bioallied Sciences, vol. 6, no. 4, pp. 233-240, 2014.

[120] S. Lindemann, M. Sharafi, M. Spiecker et al., "NO reduces PMN adhesion to human vascular endothelial cells due to downregulation of ICAM-1 mRNA and surface expression," Thrombosis Research, vol. 97, no. 3, pp. 113-123, 2000.

[121] S. Kanwar and P. Kubes, "Nitric oxide is an antiadhesive molecule for leukocytes," New Horizons, vol. 3, no. 1, pp. 93-104, 1995.

[122] K. Kasuno, S. Takabuchi, K. Fukuda et al., "Nitric oxide induces hypoxia-inducible factor 1 activation that is dependent on MAPK and phosphatidylinositol 3-kinase signaling," Journal of Biological Chemistry, vol. 279, no. 4, pp. 2550-2558, 2004.

[123] J. Mateo, M. García-Lecea, S. Cadenas, C. Hernández, and S. Moncada, "Regulation of hypoxia-inducible factor- $1 \alpha$ by nitric oxide through mitochondria-dependent and -independent pathways," Biochemical Journal, vol. 376, no. 2, pp. 537-544, 2003.

[124] E. Alchera, L. Tacchini, C. Imarisio et al., "Adenosinedependent activation of hypoxia-inducible factor-1 induces late preconditioning in liver cells," Hepatology, vol. 48, no. 1, pp. 230-239, 2008.

[125] J. A. Luciano, T. Tan, Q. Zhang, E. Huang, P. Scholz, and H. R. Weiss, "Hypoxia inducible factor-1 improves the actions of nitric oxide and natriuretic peptides after simulated ischemiareperfusion," Cellular Physiology and Biochemistry, vol. 21, no. 5-6, pp. 421-428, 2008.

[126] Z.-H. Shao, K. R. Wojcik, A. Dossumbekova et al., "Grape seed proanthocyanidins protect cardiomyocytes from ischemia and 
reperfusion injury via Akt-NOS signaling," Journal of Cellular Biochemistry, vol. 107, no. 4, pp. 697-705, 2009.

[127] K. Izuishi, A. Tsung, M. A. Hossain et al., "Ischemic preconditioning of the murine liver protects through the Akt kinase pathway," Hepatology, vol. 44, no. 3, pp. 573-580, 2006.

[128] R. Carini, M. G. De Cesaris, R. Splendore et al., "Role of phosphatidylinositol 3-kinase in the development of hepatocyte preconditioning," Gastroenterology, vol. 127, no. 3, pp. 914-923, 2004.

[129] A. Tsang, D. J. Hausenloy, M. M. Mocanu, and D. M. Yellon, "Postconditioning: a form of 'modified reperfusion' protects the myocardium by activating the phosphatidylinositol 3-kinaseAkt pathway," Circulation Research, vol. 95, no. 3, pp. 230-232, 2004.

[130] J.-S. Kim, S. Ohshima, P. Pediaditakis, and J. J. Lemasters, "Nitric oxide protects rat hepatocytes against reperfusion injury mediated by the mitochondrial permeability transition," Hepatology, vol. 39, no. 6, pp. 1533-1543, 2004.

[131] J. Roller, M. W. Laschke, C. Scheuer, and M. D. Menger, "Heme oxygenase (HO)-1 protects from lipopolysaccharide (LPS)-mediated liver injury by inhibition of hepatic leukocyte accumulation and improvement of microvascular perfusion," Langenbeck's Archives of Surgery, vol. 395, no. 4, pp. 387-394, 2010.

[132] H. Kato, F. Amersi, R. Buelow et al., "Heme oxygenase-1 overexpression protects rat livers from ischemia/reperfusion injury with extended cold preservation," American Journal of Transplantation, vol. 1, no. 2, pp. 121-128, 2001.

[133] L. Devey, D. Ferenbach, E. Mohr et al., "Tissue-resident macrophages protect the liver from ischemia reperfusion injury via a heme oxygenase-1-dependent mechanism," Molecular Therapy, vol. 17, no. 1, pp. 65-72, 2009.

[134] Z. F. Yang, T. Y. Tsui, D. W. Ho, T. C. Tang, and S.-T. Fan, "Heme oxygenase-1 potentiates the survival of small-for-size liver graft," Liver Transplantation, vol. 10, no. 6, pp. 784-793, 2004.

[135] G. Jancsó, B. Cserepes, B. Gasz et al., "Expression and protective role of heme oxygenase-1 in delayed myocardial preconditioning," Annals of the New York Academy of Sciences, vol. 1095, pp. 251-261, 2007.

[136] I.-R. Lai, K.-J. Chang, C.-F. Chen, and H.-W. Tsai, “Transient limb ischemia induces remote preconditioning in liver among rats: the protective role of heme oxygenase-1," Transplantation, vol. 81, no. 9, pp. 1311-1317, 2006.

[137] I. H. Mallick, M. C. Winslet, and A. M. Seifalian, "Ischemic preconditioning of small bowel mitigates the late phase of reperfusion injury: heme oxygenase mediates cytoprotection," The American Journal of Surgery, vol. 199, no. 2, pp. 223-231, 2010.

[138] F. Tamion, V. Richard, S. Renet, and C. Thuillez, "Intestinal preconditioning prevents inflammatory response by modulating heme oxygenase-1 expression in endotoxic shock model," American Journal of Physiology-Gastrointestinal and Liver Physiology, vol. 293, no. 6, pp. G1308-G1314, 2007.

[139] B. Xu, X. Gao, J. Xu et al., "Ischemic postconditioning attenuates lung reperfusion injury and reduces systemic proinflammatory cytokine release via heme oxygenase 1," Journal of Surgical Research, vol. 166, no. 2, pp. e157-e164, 2011.

[140] Z.-Y. Xia, J. Gao, A. K. Ancharaz, K.-X. Liu, Z. Xia, and T. Luo, "Ischaemic post-conditioning protects lung from ischaemiareperfusion injury by up-regulation of haeme oxygenase-1," Injury, vol. 41, no. 5, pp. 510-516, 2010.
[141] N. Selzner, M. Boehnert, and M. Selzner, "Preconditioning, postconditioning, and remote conditioning in solid organ transplantation: basic mechanisms and translational applications," Transplantation Reviews, vol. 26, no. 2, pp. 115-124, 2012. 


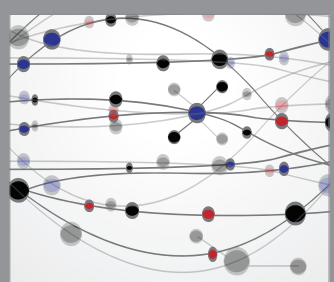

The Scientific World Journal
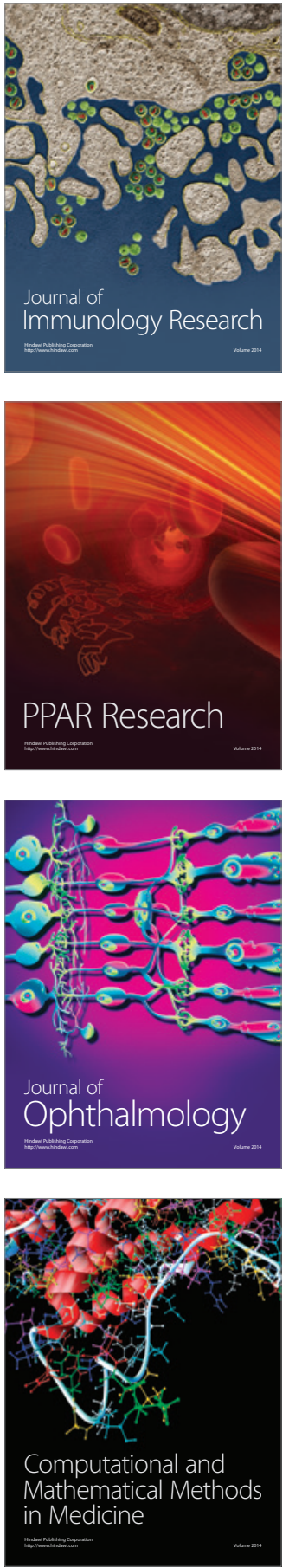

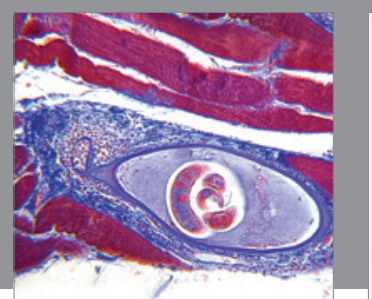

Gastroenterology Research and Practice

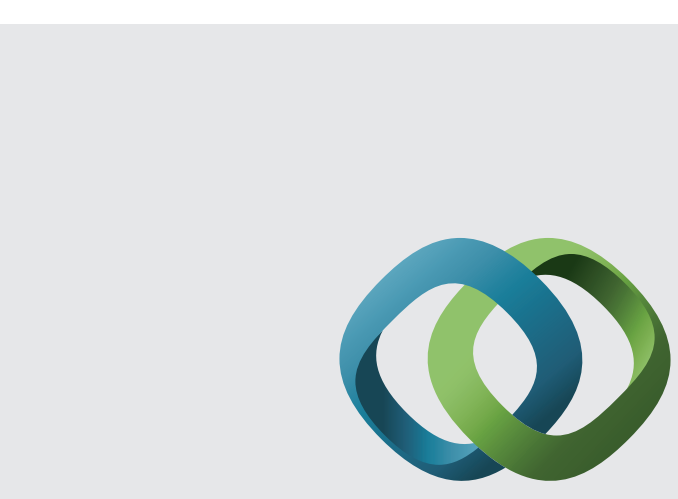

\section{Hindawi}

Submit your manuscripts at

http://www.hindawi.com
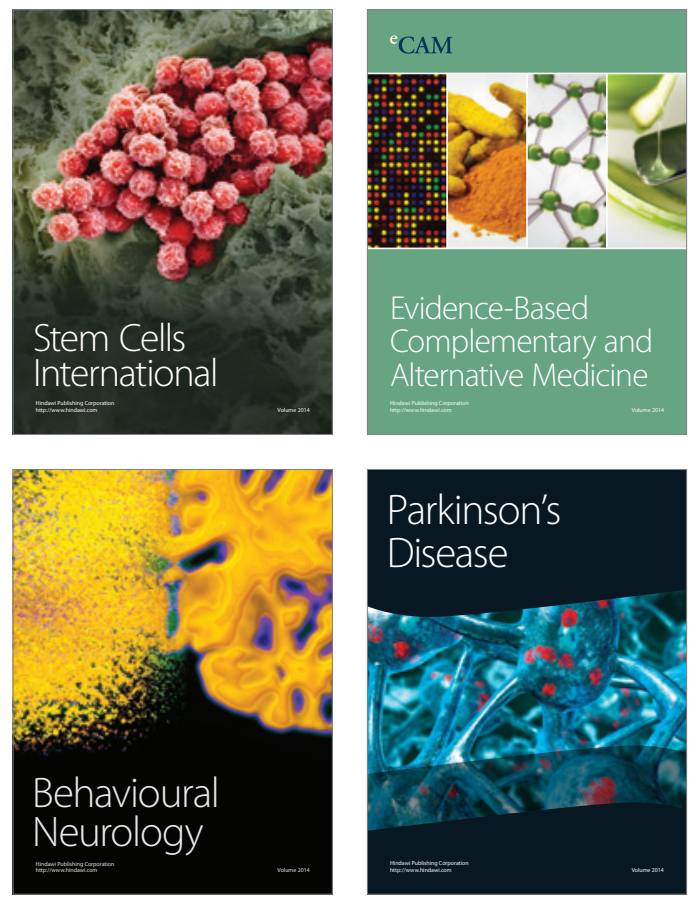
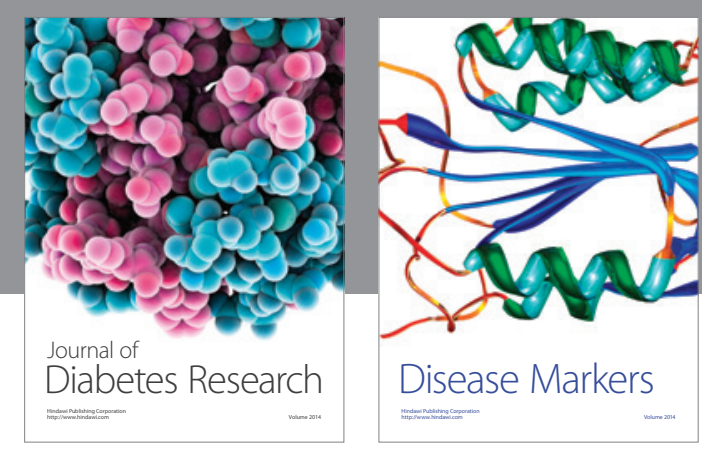

Disease Markers
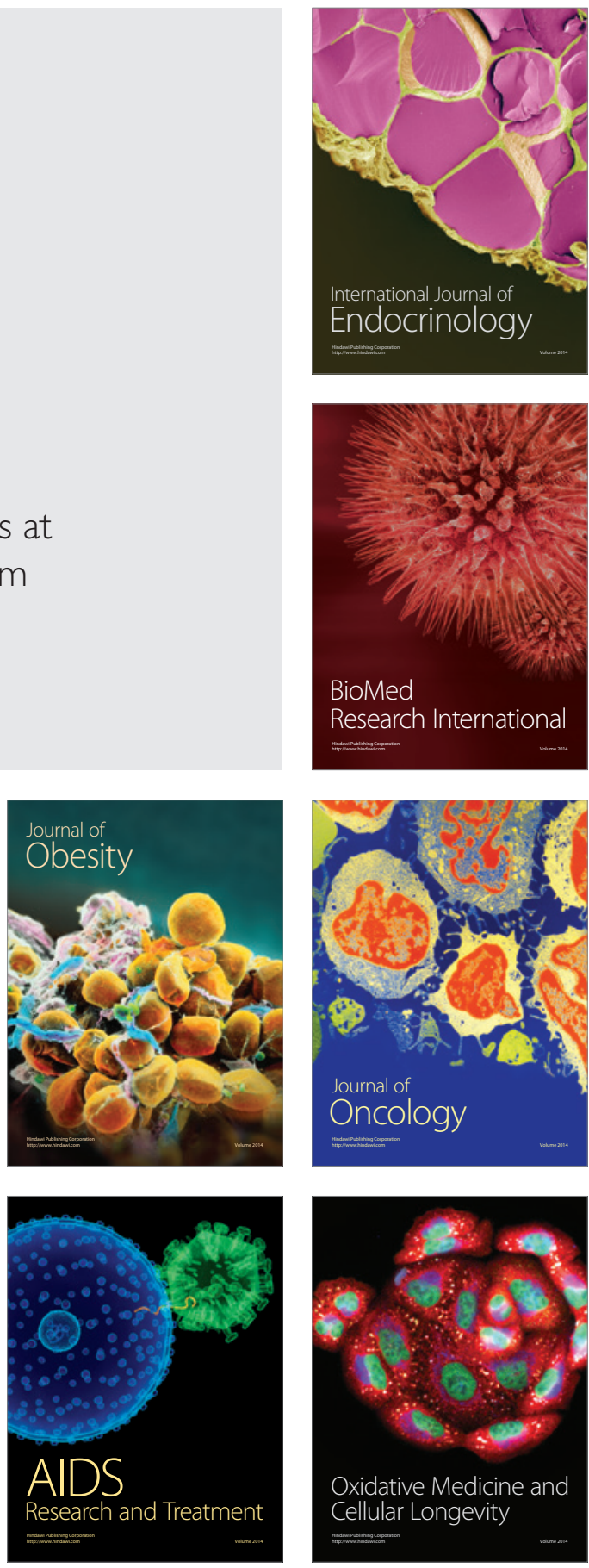